\title{
Search for flavor-changing neutral current interactions of the top quark and the Higgs boson decaying to a bottom quark-antiquark pair at $\sqrt{s}=13 \mathrm{TeV}$
}

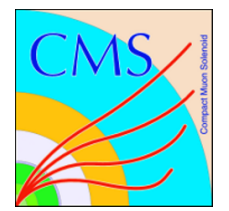

\section{The CMS collaboration}

E-mail: cms-publication-committee-chair@cern.ch

ABSTRACT: A search for flavor-changing neutral current interactions of the top quark ( $\mathrm{t}$ ) and the Higgs boson $(\mathrm{H})$ is presented. The search is based on a data sample corresponding to an integrated luminosity of $137 \mathrm{fb}^{-1}$ recorded by the CMS experiment at the LHC in proton-proton collisions at $\sqrt{s}=13 \mathrm{TeV}$. Events containing exactly one lepton (muon or electron) and at least three jets, among which at least two are identified as originating from the hadronization of a bottom quark, are analyzed. A set of deep neural networks is used for kinematic event reconstruction, while boosted decision trees distinguish the signal from the background events. No significant excess over the background predictions is observed, and upper limits on the signal production cross sections are extracted. These limits are interpreted in terms of top quark decay branching fractions $(\mathcal{B})$ to the Higgs boson and an up (u) or a charm quark (c). Assuming one nonvanishing extra coupling at a time, the observed (expected) upper limits at $95 \%$ confidence level are $\mathcal{B}(\mathrm{t} \rightarrow \mathrm{Hu})<0.079(0.11) \%$ and $\mathcal{B}(\mathrm{t} \rightarrow \mathrm{Hc})<0.094(0.086) \%$.

Keywords: B Physics, Flavour Changing Neutral Currents, Hadron-Hadron Scattering, Higgs Physics, Top Physics

ArXiv EPrint: 2112.09734 


\section{Contents}

1 Introduction 1

2 The CMS detector 3

3 Signal and background modeling 3

4 Event reconstruction and selection 4

5 Signal extraction $\quad 6$

6 Systematic uncertainties $\quad 8$

$\begin{array}{lll}7 & \text { Results } & 12\end{array}$

$\begin{array}{lll}8 & \text { Summary } & 14\end{array}$

$\begin{array}{ll}\text { The CMS collaboration } & 20\end{array}$

\section{Introduction}

The discovery of a new scalar boson with properties compatible with the standard model (SM) Higgs boson $(\mathrm{H})[1-4]$ opened a new window to shed light on beyond-the-SM phenomena at the CERN LHC. The top quark (t) is the most massive known fermion and its measured Yukawa coupling is of order unity [5]. The large mass of the top quark $\left(m_{\mathrm{t}}\right)$ suggests that it may play an important role in electroweak symmetry breaking [6-8]. Since the Yukawa coupling of the Higgs boson to fermions is proportional to the mass of the fermion, it is of particular importance to study the coupling between the top quark and Higgs boson.

Flavor-changing neutral current (FCNC) interactions of the Hqt vertex (where q represents quarks) are key processes to search for new physics. The FCNC process is forbidden at tree level in the SM and is highly suppressed in loop corrections by the GlashowIliopoulos-Maiani mechanism [9], leading to a branching fraction of $\mathcal{O}\left(10^{-17}\right)$ for $\mathrm{t} \rightarrow \mathrm{Hu}$ and $\mathcal{O}\left(10^{-15}\right)$ for $\mathrm{t} \rightarrow \mathrm{Hc}[10-12]$ with the up (u) and charm quark (c) final states, respectively. On the contrary, many SM extensions, such as the minimal supersymmetric standard model [13], supersymmetric models with R-parity violation [14], and other twoHiggs-doublet models [15-17], predict highly enhanced $t \rightarrow$ Hq branching fractions $[13,17-$ 20]. Values can be as high as $\mathcal{O}\left(10^{-5}\right)$ for $\mathrm{t} \rightarrow \mathrm{Hu}$ and $\mathcal{O}\left(10^{-3}\right)$ for $\mathrm{t} \rightarrow \mathrm{Hc}$ [10], which are within the experimental sensitivity of analyses performed with the LHC data at $13 \mathrm{TeV}$.

Several searches for anomalous couplings between the top quark and the Higgs boson have recently been carried out by the ATLAS [21-23] and CMS [24, 25] Collaborations. The 

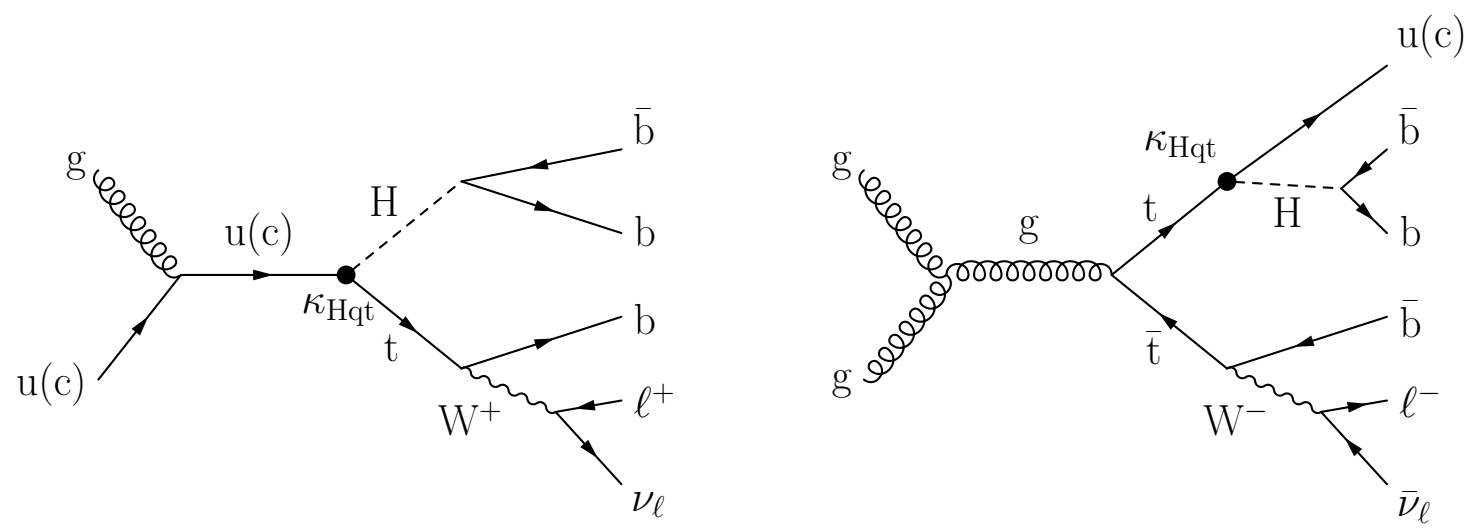

Figure 1. Illustrative Feynman diagrams for Hqt FCNC interactions: the production of a single top quark with the Higgs boson (left), and FCNC decay of the top quark in t $\bar{t}$ events (right), where $\mathrm{q}=\mathrm{u}$ or $\mathrm{c}$.

most recent analysis is performed by CMS using a data sample with an integrated luminosity of $137 \mathrm{fb}^{-1}$ in the diphoton decay channel of the Higgs boson, yielding observed limits on the branching fractions of 0.019 and $0.078 \%$ for $\mathrm{t} \rightarrow \mathrm{Hu}$ and $\mathrm{t} \rightarrow \mathrm{Hc}$, respectively [25].

In this paper, we present an extension of the CMS search for Hqt FCNC interactions in the $\mathrm{H} \rightarrow \mathrm{b} \overline{\mathrm{b}}$ final state [24], exploiting a $13 \mathrm{TeV}$ data set corresponding to an integrated luminosity of $137 \mathrm{fb}^{-1}$. Proton-proton (pp) collision data collected by CMS during 2017 and $2018\left(101 \mathrm{fb}^{-1}\right)$ are analyzed separately and combined with the earlier results in the $\mathrm{H} \rightarrow \mathrm{b} \overline{\mathrm{b}}$ channel based on the 2016 data-taking period. Events are selected in the final state with exactly one lepton $(\ell=\mu$ or e) and at least three jets, of which at least two must be identified as coming from the hadronization of a bottom quark (b jets). Two signal production modes arising from the anomalous Hqt interaction are considered: the associated production of a single top quark with the Higgs boson from an u or c quark (ST production mode) and the $\mathrm{t} \rightarrow \mathrm{Hq}$ FCNC decays of a top quark in top quark-antiquark pair $(\mathrm{t} \overline{\mathrm{t}})$ events (TT production mode), as presented in figure 1. In addition to the increased integrated luminosity, the analysis utilizes advanced techniques including a deep neural network (DNN) and boosted decision trees (BDTs) to improve the performance of event reconstruction and signal extraction, and in the $\mathrm{H} \rightarrow \mathrm{b} \overline{\mathrm{b}}$ final state it places the most stringent limits to date on the $\mathrm{t} \rightarrow \mathrm{Hu}$ and $\mathrm{t} \rightarrow \mathrm{Hc}$ branching fractions. Tabulated results are provided in the HEPData record for this search [26].

The paper is organized as follows. We begin with a brief overview of the CMS detector in section 2, followed by a description of the signal and background modeling in section 3 . The event selection and signal extraction using multivariate analysis techniques are detailed in sections 4 and 5, respectively. Numerous systematic uncertainties contribute to the analysis - these are explained in section 6. Finally, the results of the search are presented in section 7 and we conclude with a summary in section 8 . 


\section{The CMS detector}

The central feature of the CMS apparatus is a superconducting solenoid of $6 \mathrm{~m}$ internal diameter, providing a magnetic field of $3.8 \mathrm{~T}$. Within the solenoid volume, there are a silicon pixel and strip tracker, a lead tungstate crystal electromagnetic calorimeter (ECAL), and a brass and scintillator hadron calorimeter (HCAL), each composed of a barrel and two endcap sections. Forward calorimeters extend the pseudorapidity $(\eta)$ coverage provided by the barrel and endcap detectors. Muons are detected in gas-ionization chambers embedded in the steel flux-return yoke outside the solenoid. Events of interest are selected using a twotiered trigger system. The first level is composed of custom hardware processors and selects events at a rate of around $100 \mathrm{kHz}$ [27]. The second level, known as the high-level trigger, is implemented in software running on a processor farm and reduces the event rate to around $1 \mathrm{kHz}$ before data storage [28]. A more detailed description of the CMS detector, together with a definition of the coordinate system used and the relevant kinematic variables, can be found in ref. [29].

\section{$3 \quad$ Signal and background modeling}

The signal samples are generated at leading order (LO) in perturbative quantum chromodynamics (QCD) with MADGRAPH5_amC@NLO 2.6.0 (TT production mode) or 2.4.2 (ST production mode) [30]. The Lagrangian with a FCNC coupling $\left(\kappa_{\mathrm{Hqt}}\right)$

$$
\mathcal{L}=\sum_{\mathrm{q}=\mathrm{u}, \mathrm{c}} \frac{g}{\sqrt{2}} \overline{\mathrm{t}} \kappa_{\mathrm{Hqt}}\left(f_{\mathrm{Hq}}^{\mathrm{L}} P_{\mathrm{L}}+f_{\mathrm{Hq}}^{\mathrm{R}} P_{\mathrm{R}}\right) \mathrm{q} \mathrm{H}+\text { h.c. }
$$

is implemented in the FEYNRULES package [31], and the universal FEYNRULES output format [32] is used to generate the signal model. The complex chiral parameters $f_{\mathrm{Hq}}^{\mathrm{L}}$ and $f_{\mathrm{Hq}}^{\mathrm{R}}$ have negligible impact on the kinematics, and are fixed to 0 and 1 , respectively. Up to two additional partons are generated at the matrix-element level for the TT production mode, while none are considered for the ST mode as they would induce an overlap between the two processes. The MLM [33] matching prescription is used to interface the hard-process simulation with the parton-shower modeling. The ST and TT processes are generated for two scenarios (Hut or Hct), assuming the presence of only one nonvanishing FCNC coupling at a time $\left(\kappa_{\mathrm{Hut}}\right.$ or $\left.\kappa_{\mathrm{Hct}}\right)$. The TT signal production cross section predicted to $46.5 \mathrm{pb}$ for each coupling, obtained by multiplying the SM t $\overline{\mathrm{t}}$ production cross section by the corresponding branching fraction of the $\mathrm{t} \rightarrow \mathrm{Hq}$ decay, computed by MADGRAPH5_aMC@NLO. The predicted $t \overline{\mathrm{t}}$ production cross section is $832 \mathrm{pb}$, as calculated with the TOP ++ program at next-to-next-to-LO (NNLO) in perturbative QCD including soft-gluon resummation to next-to-next-to-leading-logarithmic order [34]. The cross section of the subdominant ST production mode is 13.8 (1.9) pb when setting the Hut (Hct) coupling to unity, computed by MADGRAPH5_aMC@NLO with LO precision. It is known that the top quark transverse momentum $\left(p_{\mathrm{T}}\right)$ spectra in $\mathrm{t} \overline{\mathrm{t}}$ production using LO and next-to-LO (NLO) predictions differ from the higher-order theoretical prediction. The distribution and normalization of the top quark $p_{\mathrm{T}}$ spectrum in the TT signal events are therefore corrected first from LO to 


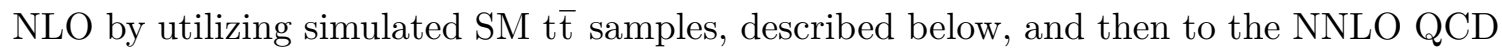
+ NLO electroweak prediction $[35,36]$.

The dominant background process is SM têt production, which is simulated at NLO precision using POWHEG v2 [37-39]. The $t \bar{t}$ events are categorized based on the flavor of the additional particle-level jets (those not originating from the top quark decays): events where at least one additional $b$ jet is present $(t \bar{t} b \bar{b})$; events where at least one additional $c$ jet is present that are not categorized as $t \bar{t} b \bar{b}$ events $(t \bar{t} c \bar{c})$; and other events ( $t \bar{t} L F$, where LF stands for light flavor). Similar to the TT signal samples, the $p_{\mathrm{T}}$ spectrum in the SM t $\overline{\mathrm{t}}$ sample is corrected from the NLO to the higher-order prediction. In addition, the single-leptonic and dileptonic decays of the SM t $\bar{t} \mathrm{~b} \overline{\mathrm{b}}$ process are corrected for observed underestimations in simulation of about $20 \%$ [40], and the corresponding uncertainties derived from the measurement are assigned. The subdominant single top quark background is considered in the $t$ channel, $s$ channel, and in association with a $\mathrm{W}$ boson (tW). The $t$-channel production is simulated using POWHEG v2, while the $s$-channel production is generated with MADGRAPH5_aMC@NLO and tW samples are obtained from POWHEG v1 [41]. The predicted NLO cross sections are $136(81)$ pb for $t$-channel top quark (antiquark) production and $10.3 \mathrm{pb}$ for $s$-channel production [42,43]. The tW cross section considered

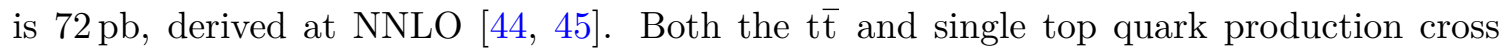
sections are calculated assuming $m_{\mathrm{t}}=172.5 \mathrm{GeV}$. The uncertainties associated with these predictions are described in section 6. Smaller SM background contributions arise from the following processes: $t \bar{t}$ production in association with a vector boson ( $t \bar{t} Z$ and $t \bar{t} \mathrm{~W}$ ) or a Higgs boson ( $\mathrm{t} \overline{\mathrm{t}} \mathrm{H})$, simulated with MADGRAPH5_aMC@NLO and POWHEG v1, respectively; Drell-Yan and W boson production, simulated with MADGRAPH5_aMC@NLO; and diboson (VV) processes generated with PYTHIA 8.230 [46]. In addition, multijet events are simulated with PYтнIA 8.230 and used in the phase space where the estimated multijet background is nonnegligible.

The parton distribution function (PDF) set used for the event generation is NNPDF3.1 [47], while the handling of the initial- and final-state radiation, the parton showering, and the hadronization is performed by PYTHIA 8.230 with the CP5 tune [48]. Additional inelastic pp interactions within the same or nearby bunch crossings (pileup) are simulated using PYTHIA and added to the generated process of interest. The number of such interactions to be added is estimated based on the measured instantaneous luminosity and a total pp inelastic cross section of $69.2 \mathrm{mb}$ [49]. The events are corrected to remove differences between the simulated and observed pileup distributions. All the outgoing particles are propagated through a detailed simulation of the CMS detector based on the GEANT4 [50] framework, which models the interactions with matter, the energy deposits in sensitive detector layers, and the resulting electronic responses.

\section{Event reconstruction and selection}

The particle-flow (PF) algorithm [51] aims to reconstruct and identify each individual particle in an event, with an optimized combination of information from the various elements of the CMS detector. The reconstructed vertex with the largest value of summed 
physics-object $p_{\mathrm{T}}^{2}$ is taken to be the primary pp interaction vertex. The energy of electrons is determined from a combination of the electron momentum at the primary interaction vertex as determined by the tracker, the energy of the corresponding ECAL cluster, and the energy sum of all bremsstrahlung photons spatially compatible with originating from the electron track. The muon $p_{\mathrm{T}}$ is obtained from the curvature of the corresponding track built using the tracker and muon chambers. The energy of charged hadrons is determined from a combination of their momentum measured in the tracker and the matching ECAL and HCAL energy deposits calibrated by the response function of the calorimeters to hadronic showers. The energy of neutral hadrons is obtained from the corresponding corrected ECAL and HCAL energies. Finally, the missing transverse momentum vector $\vec{p}_{\mathrm{T}}^{\text {miss }}$ is computed as the negative vector $\vec{p}_{\mathrm{T}}$ sum of all the PF candidates in an event, and its magnitude is denoted as $p_{\mathrm{T}}^{\text {miss }}[52]$.

Jets are reconstructed from the PF candidates using the anti- $k_{\mathrm{T}}$ clustering algorithm $[53,54]$ with a distance parameter of 0.4 . The measured momentum in simulation agrees within $5-10 \%$ of the true momentum over the entire $p_{\mathrm{T}}$ spectrum and detector acceptance [55]. To mitigate the pileup effect on the jet momentum, tracks identified to be originating from pileup vertices are discarded and an offset correction is applied to correct for remaining contributions. Jet energy corrections are derived from simulation and confirmed with in situ measurements of the energy balance in dijet, multijet, $\gamma+$ jet, and leptonic $\mathrm{Z}+$ jet events. Only jets with $p_{\mathrm{T}}>30 \mathrm{GeV}$ and $|\eta|<2.4$ are considered and an additional selection is applied to remove spurious jet-like features originating from isolated noise patterns in certain HCAL regions [56]. Heavy-flavor jets are identified based on the DeepCSV algorithm [57] at a working point characterized by a b-jet identification efficiency of $68 \%$, a c-jet and a light-quark or gluon jet misidentification rate of 12 and 1\%, respectively. An event-by-event correction based on the jet flavor content is applied to account for differences between the simulated and observed DeepCSV distributions [57]. The analysis requires the presence of at least three jets, among which two or more must be identified as b jets.

The analyzed events were recorded using a combination of muon and electron triggers. Events in the muon channel are selected using a trigger requiring at least one muon with $p_{\mathrm{T}}>27(24) \mathrm{GeV}$ for the 2017 (2018) data-taking period; in the electron channel, triggers used require either at least one electron with $p_{\mathrm{T}}>35(32) \mathrm{GeV}$ or at least one electron with $p_{\mathrm{T}}>28 \mathrm{GeV}$ and $|\eta|<2.1$, as well as a global scalar sum of jet transverse momenta exceeding $150 \mathrm{GeV}$. The corrections to the muon trigger efficiencies are derived using the "tag-and-probe" technique [58] in a data sample enriched with Z $\rightarrow \ell \ell$ events. To calculate the electron trigger efficiencies in a similar way without bias, orthogonal singlemuon data sets are used together with t $\bar{t}$ events in the single-lepton and dilepton channels. Muon candidates are selected offline based on the track fit quality, the number of hits associated with the track, and the track impact parameter [59]. For electron candidates, offline requirements on the shower shape and track identification are applied [60]. The analysis only considers leptons with $p_{\mathrm{T}}>30 \mathrm{GeV}$ and $|\eta|<2.4$. Additionally, electrons reconstructed in the gap between the ECAL barrel and endcap $(1.44<|\eta|<1.57)$ are rejected. To reduce the contribution from leptonic decays of heavy-flavor hadrons, a selection on the isolation parameter $I_{\text {rel }}$ is applied. This variable is defined as the ratio of the 
scalar $p_{\mathrm{T}}$ sum of photons, charged and neutral hadrons within a cone of angular distance $\Delta R=\sqrt{(\Delta \eta)^{2}+(\Delta \phi)^{2}}=0.4(0.3)$ for muons (electrons) to the lepton $p_{\mathrm{T}}$. The muon candidates are required to have $I_{\text {rel }}<0.15$ while for electron candidates the requirement is defined in bins of $p_{\mathrm{T}}$ and $\eta$. Events with additional leptons, defined using looser identification criteria, are rejected to reduce background contributions containing nonprompt leptons.

\section{Signal extraction}

The selected events are divided into five categories based on the jet and b-tagged jet multiplicities in order to optimize the analysis sensitivity. The number of jets can be exactly three or at least four, and the corresponding categories are denoted as $\mathrm{j} 3 \mathrm{or} \mathrm{j} 4$, respectively. The events with up to four b jets are analyzed, considering misidentification of jets and additional partons in the matrix-element calculation. Similar to the jet multiplicity, the categories in terms of $\mathrm{b}$ jets are indicated as b2, b3, or b4 when the considered event has exactly two, three, or four b jets, respectively. Events with five or more b jets are not used due to their large uncertainty and small impact on the analysis. Following these conventions, the jet categories are identified as $\mathrm{b} 2 \mathrm{j} 3, \mathrm{~b} 2 \mathrm{j} 4, \mathrm{~b} 3 \mathrm{j} 3, \mathrm{~b} 3 \mathrm{j} 4$, and $\mathrm{b} 4 \mathrm{j} 4$. The analysis follows two steps of multivariate techniques to identify signal events over the SM prediction. Considering each jet category separately, events are reconstructed using a set of DNNs, one for each relevant signal and background process hypothesis. For each process hypothesis, the DNN selects the best combination of jets in each event. Thus, a given event may have more than one jet assignment, depending on the available process hypothesis. The BDTs are then utilized for signal event discrimination against the SM backgrounds using the reconstructed kinematic variables from the jet combinations from all possible process hypotheses. The obtained distributions for the simulated samples are then fit to the data using a binned maximum likelihood fit.

To maximize the sensitivity to the signal processes and to discriminate against the SM backgrounds, it is necessary to fully characterize the signal and background kinematic properties and gather discriminative features. Thus, one has to correctly assign the reconstructed jets to the generator-level jets in the signal or background simulated samples to build the best possible distributions of the kinematic variables. The event reconstruction is performed under multiple process hypotheses, namely the ST and TT signal hypotheses and the SM t $\overline{\mathrm{t}}$ background hypothesis. Each hypothesis can be distinguished by the number of jets in the final state and their flavor, without considering jets from additional partons or radiation. The signal hypotheses commonly contain three $b$ jets from the Higgs boson and the leptonically decaying top quark, and the TT signal process has an additional up or charm quark jet. For the SM t $\bar{t}$ hypothesis, there is one $b$ jet from each top quark decay and two additional jets from the hadronic decay of a $\mathrm{W}$ boson. Consequently, the ST hypothesis is considered for events with three or more jets, while the TT and t $\overline{\mathrm{t}}$ hypotheses are applied to the events with four or more jets.

Each reconstruction algorithm is constructed as a binary classifier DNN with several possible combinations of three (ST) or four (TT, t $\bar{t}$ ) jets in the event as inputs. For each event, the signal combination is defined as the case in which all reconstructed jets in the 
combination are matched to the generator-level jets within an angular distance of 0.4. All the other combinations are labeled as background. To reduce the number of wrong combinations considered, the TT and ST signal hypothesis training for events with two (at least three) $b$ jets is performed only for configurations in which the Higgs boson is associated with at least one (two) b jet(s). After optimization of the performance, and taking into account agreement between data and simulation, 32 (69) kinematic variables are constructed for the ST (TT and SM t $\overline{\mathrm{t}}$ ) hypotheses and used for the training, such as the angular distance, momentum, and $\eta$ of jets, dijets, or trijet combinations as well as b tagging discriminants.

A residual network structure $[61,62]$ is chosen to boost training speed and to prevent information loss that may occur for structures with a large number of hidden layers. In comparison to a simple DNN with the same number of hidden layers, the training of the residual network converges to its best performance more quickly, by up to $50 \%$, for this analysis. The residual network consists of several residual blocks as well as input and output layers. In the residual block, the input data is branched off into two streams, where one stream is passed through two hidden layers while the other bypasses them. The two streams are then added into one tensor and fed into the next residual block. The network consists of 11 hidden layers in total, with five residual blocks.

The classifiers are first trained with all jet categories for the 2017 and 2018 simulated samples, separately. In addition, an exclusive set of classifiers are trained using only the jet permutations from the $\mathrm{b} 4 \mathrm{j} 4$ category because of the limited size for the signal combinations and the large difference between the signal and background sample sizes. Once the classifiers are trained with the signal and background combinations, all combinations from the statistically independent data and simulated samples are predicted with the trained classifiers. The combination with the highest DNN score is selected for each event and process hypothesis. The performance of the classifier is measured in terms of the reconstruction efficiency, defined as the ratio of the number of events where all jets in the selected combination are correctly assigned to the number of events where the reconstructed jets are fully matched to generated jets within an angular distance of 0.4 . The computed efficiencies for the ST signal hypothesis are $\approx 86 \%$ and $\approx 82 \%$ for the Hut and Hct couplings, respectively. In the TT signal scenario, the classifier trained with the Hut (Hct) coupling shows an $\approx 79(\approx 77 \%)$ efficiency, while for the SM t $\overline{\mathrm{t}}$ hypothesis it is $\approx 78 \%$. By implementing the DNN, the reconstruction efficiencies are improved by $5-15 \%$ compared to the kinematic fit method that was used in the 2016 data analysis [24]. While the efficiencies of the exclusive training in the b4j4 category are comparable to the inclusive ones for the $\mathrm{ST}$ and $\mathrm{TT}$ hypotheses, in this phase space the $t \overline{\mathrm{t}}$ reconstruction efficiency is improved by more than $40 \%$. The efficiencies are computed with statistically independent samples with respect to the training ones. The improvements in the reconstruction algorithms benefit the analysis by helping to accurately construct the kinematic distributions. Example kinematic distributions for different process hypotheses are presented in figure 2 .

To distinguish signal events from the SM background contributions, a BDT is used for each signal coupling, jet category, and data-taking period (2017 and 2018). The ST and TT FCNC events are considered jointly as signal in the training, taking into account their relative contributions in the event yield. Among the background processes, only the dominant 

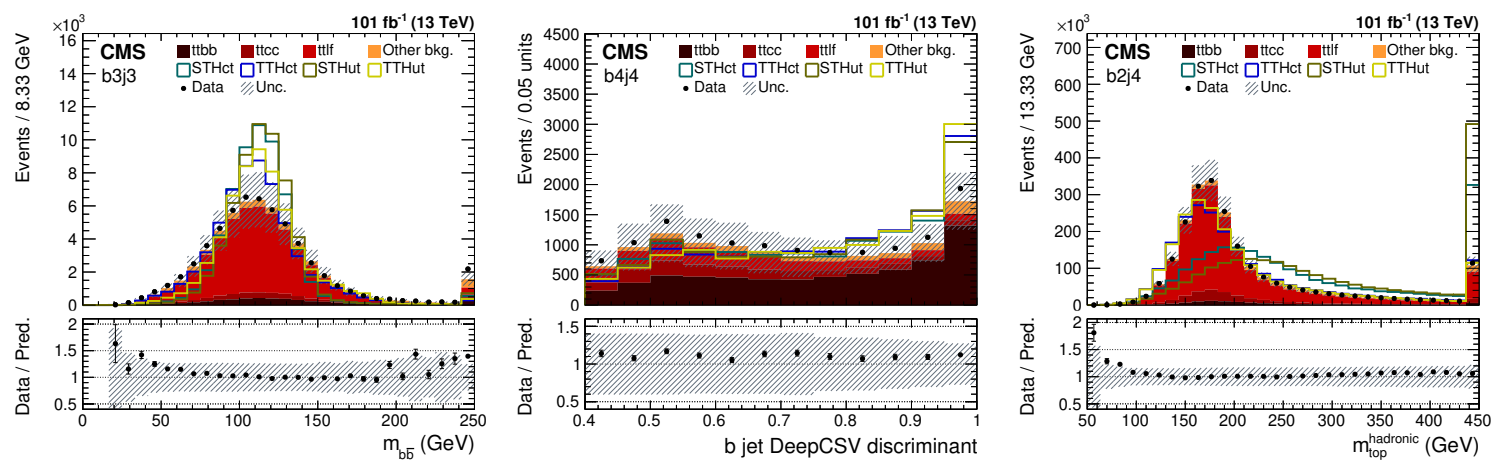

Figure 2. The reconstructed $m_{\mathrm{b} \overline{\mathrm{b}}}$ (Higgs boson candidate mass) in the b3j3 category for the ST signal scenario (left), the distribution of the second largest DeepCSV value for the b-tagged jet from the Higgs boson decay in the b4j4 category for the TT signal scenario (middle), and the mass of the hadronically decaying top quark in the b2j4 category for the $\mathrm{SM} t \overline{\mathrm{t}}$ background scenario (right) for the combined $2017+2018$ data. The lower panel shows the ratio of observed data to the SM prediction. The shaded band corresponds to the total uncertainty in the predicted background. For the mass plots the last bin contains the overflow events.

SM tE process is considered in the BDT training. A total of 164 input kinematic variables sensitive to the different process hypotheses, such as the $\mathrm{b}$ tagging discriminator scores of the selected jets or the angular distributions between particles, are used in the BDT training. Throughout the BDT training, b tagging discriminator values are found to be the most important features. For example, the distributions of the second largest DeepCSV values from the reconstructed Higgs boson in the ST and TT signal hypotheses are well separated from the SM predictions (see figure 2), as the corresponding jet typically does not

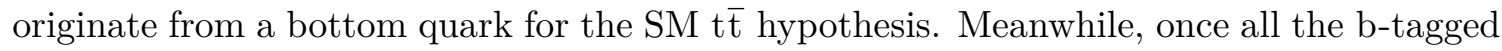
jets are assigned to the $\mathrm{b}$ jets from the SM top quarks in the SM t $\overline{\mathrm{t}}$ hypothesis, the largest DeepCSV value from the reconstructed W boson is typically larger in ST and TT events compared to the SM events. In addition, the mass distributions are also important features. If there are more than three b-tagged jets so that there are combinatorial jet permutations, the mass distribution of the reconstructed Higgs boson in FCNC events are distinguishable from the SM backgrounds. Furthermore, the mass distribution of the hadronically decaying top quark in ST events is well separated from the background as the latter contains no such top quark. In the b2j3 category, the BDT scores are additionally evaluated for the multijet process as they have nonnegligible contributions to the event yield. The post-fit BDT output distributions for different jet categories for the 2017 and 2018 data-taking periods are obtained from the binned maximum likelihood fit to the data performed with the combined 2017 and 2018 data and simulation, and presented in figures 3 (Hut) and 4 (Hct coupling).

\section{Systematic uncertainties}

The impact of experimental and theoretical systematic uncertainties is evaluated by means of nuisance parameters, described further in section 7. The dominant source of experimental uncertainty comes from the imperfect knowledge of the $\mathrm{b}$ tagging (mis-)identification rate, which affects both the event yield in each analysis category and the discriminant distri- 

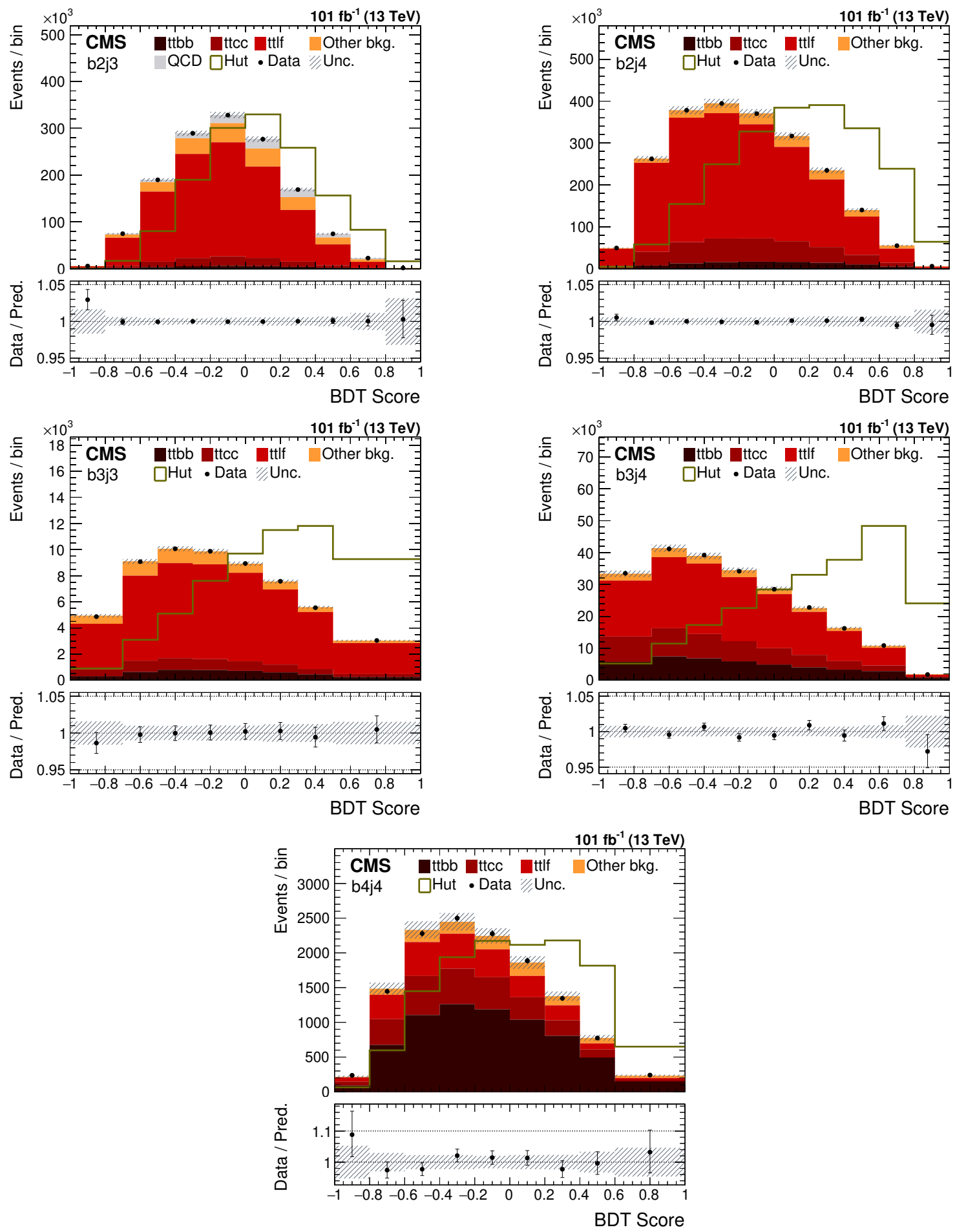

Figure 3. The BDT output distributions for the combined $2017+2018$ data and simulation for the different jet categories, assuming the Hut coupling. The lower panel shows the ratio of observed data to the SM prediction. The shaded bands correspond to the post-fit total uncertainty in the predicted background. The signal contributions are normalized to the total number of events in data. 

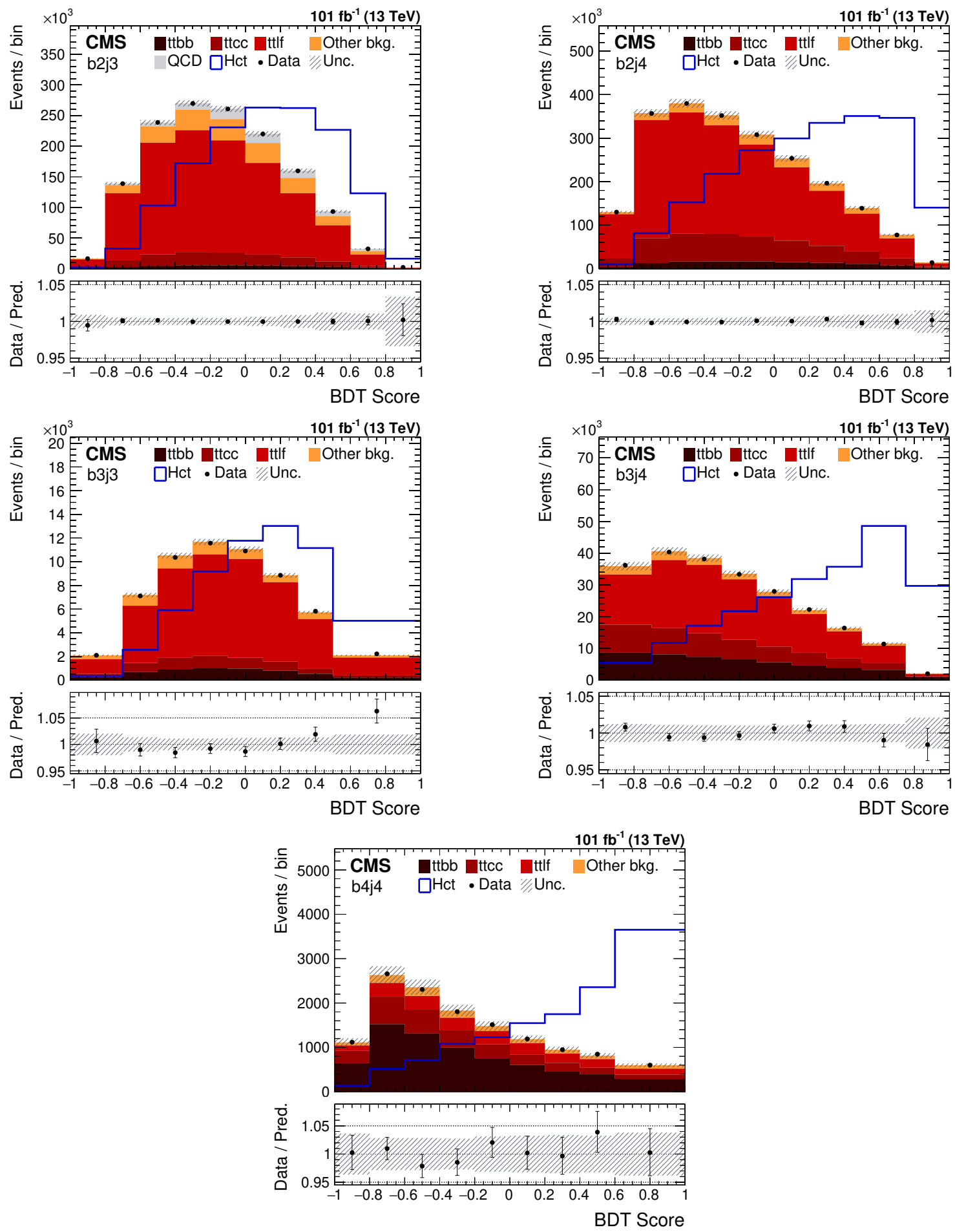

Figure 4. The BDT output distributions for the combined 2017+2018 data and simulation for the different jet categories, assuming the Hct coupling. The lower panel shows the ratio of observed data to the SM prediction. The shaded bands correspond to the post-fit total uncertainty in the predicted background. The signal contributions are normalized to the total number of events in data. 
butions. Its magnitude is evaluated separately for $\mathrm{b}$ jets and light-quark or gluon jets, and is derived based on a $t \bar{t}(\mathrm{Z})$ enriched sample for the former (latter). The total magnitude of the $\mathrm{b}$ tagging uncertainty varies with the jet category and ranges from 12 to $25 \%$.

The uncertainty arising from the jet energy scale (JES) and resolution (JER) also impacts both the distributions and the event yields for the signal and background processes. The JES uncertainties are calculated by splitting into each one of the uncertainty sources, such as pileup, detector response, discrepancies in jet $p_{\mathrm{T}}$ and $\eta$, and flavor. The uncertainties are then grouped into seven sources defined according to their correlations and detector geometry. The uncertainty induced by the JER is assessed for different sources, such as the statistical uncertainty in the JER measurement, jet radiation, underlying event, out-of-cone showering, and pileup. A detailed description of the determination of these uncertainties can be found in ref. [55]. The uncertainties in the muon and electron scale factors related to their identification, isolation, and trigger efficiency are assessed when deriving these scale factors using the tag-and-probe technique, taking various systematic sources into account, including the limited size of the sample used, the possible bias from the tag selection, as well as run-by-run differences. The experimental uncertainties in the integrated luminosity measurements for the data recorded in 2017 and 2018, respectively, are estimated to be 2.3 and $2.5 \%$ [63, 64], while the integrated luminosity of the combined 2017-2018 data set has an uncertainty of $2.0 \%$. To account for the uncertainty in the pileup reweighting in the simulated events, the value of the total pp inelastic cross section, which is used to estimate the mean number of additional pp interactions, is varied by $\pm 4.6 \%$ [49].

The following sources of theoretical uncertainty are taken into account. The leading source of theoretical uncertainties is coming from the arbitrary choice of renormalization and factorization scales and is obtained by taking the envelope of the distributions obtained with modified scales $[65,66]$. The scale variation uncertainty ranges between $10-17 \%$ and constitutes the second largest uncertainty source. The uncertainties in the initial- and finalstate radiation are computed by varying the scale used to evaluate $\alpha_{\mathrm{S}}$ for each radiation by factors of 0.5 and 2 . The matching of matrix-element partons and parton showers is controlled in POWHEG by a model parameter $h_{\text {damp }}$. The nominal value for the $h_{\text {damp }}$ parameter is set to $1.379 m_{\mathrm{t}}$, with $m_{\mathrm{t}}=172.5 \mathrm{GeV}$ [48]. The uncertainty associated with this prescription is estimated by varying the $h_{\text {damp }}$ value by $0.8738 m_{\mathrm{t}}$ and $2.305 m_{\mathrm{t}}$. The impact of the uncertainty in the PDF is assessed by using various event weights that represent the usage of the uncertainty eigenvector sets of the PDF [67]. Dedicated samples

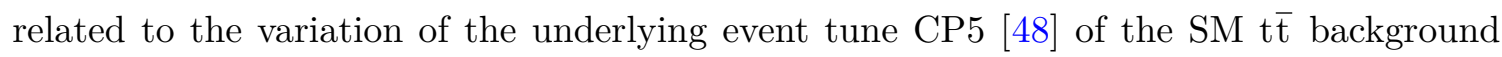
sample are used to estimate the associated uncertainty.

The calculation of the SM t $t \bar{t}$ production cross section has an uncertainty of $5.5 \%$ [37-

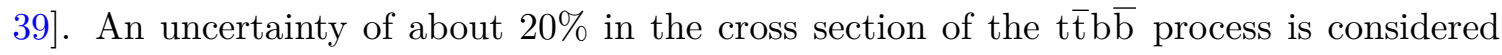
to account for the correction of the underestimated simulation [40], and an uncertainty of $50 \%$ in the cross section of the $t \overline{\mathrm{t}} \mathrm{c} \overline{\mathrm{c}}$ process is included to cover the possible discrepancy between data and simulation. The uncertainties in the normalization of the other SM background yields have been found to be subdominant and are fixed to $10 \%$. The uncertainty coming from the limited size of the simulated samples is also considered as a source of systematic uncertainty by assigning a single nuisance parameter that is constrained by the total uncertainty in each bin of the BDT output distribution [68, 69]. 


\begin{tabular}{|cccccc|}
\hline Category & $\mathrm{b} 2 \mathrm{j} 3$ & $\mathrm{~b} 2 \mathrm{j} 4$ & $\mathrm{~b} 3 \mathrm{j} 3$ & $\mathrm{~b} 3 \mathrm{j} 4$ & $\mathrm{~b} 4 \mathrm{j} 4$ \\
\hline Data & 1431931 & 2207121 & 59002 & 228311 & 12981 \\
$\mathrm{t} \overline{\mathrm{t}} \mathrm{b} \overline{\mathrm{b}}$ & $33038 \pm 6500$ & $115300 \pm 24000$ & $5452 \pm 430$ & $49251 \pm 3900$ & $6974 \pm 710$ \\
$\mathrm{t} \overline{\mathrm{t}} \overline{\mathrm{C}}$ & $115968 \pm 19000$ & $397302 \pm 61000$ & $5362 \pm 840$ & $45055 \pm 6500$ & $2815 \pm 830$ \\
$\mathrm{t} \overline{\mathrm{t}} \mathrm{LF}$ & $1020955 \pm 22000$ & $1547909 \pm 47000$ & $42893 \pm 990$ & $120172 \pm 4500$ & $2118 \pm 230$ \\
Other & $188582 \pm 17000$ & $146711 \pm 14000$ & $5508 \pm 630$ & $13757 \pm 1400$ & $1061 \pm 120$ \\
$\mathrm{QCD}$ & $73167 \pm 13000$ & - & - & - & - \\
Total & $1431710 \pm 8900$ & $2207222 \pm 13000$ & $59215 \pm 750$ & $228235 \pm 2500$ & $12967 \pm 400$ \\
\hline
\end{tabular}

Table 1. Number of events in the combined $2017+2018$ data and prediction for the backgrounds assuming absence of the signal events, shown separately for each jet category, with uncertainties obtained from the fit on the BDT distributions of Hct coupling.

\section{Results}

The BDT output distributions in figures 3 and 4 show no significant excess with respect to the SM background expectations. Upper limits at 95\% confidence level (CL) are placed on the product of the signal production cross section and branching fraction. The limits are derived based on the asymptotic modified frequentist method (asymptotic $\mathrm{CL}_{\mathrm{s}}$ ) with the test statistic based on the profile likelihood ratio consists of binned likelihood as a function of signal strength and nuisance parameters [70-72], separately for the $\mathrm{t} \rightarrow \mathrm{Hu}$ and $\mathrm{t} \rightarrow \mathrm{Hc}$ couplings, i.e., assuming one nonvanishing coupling at a time. All the systematic uncertainties described in section 6 are considered as nuisance parameters in the fit. The uncertainty sources affecting the global normalization are considered with log-normal probability distributions, while the ones affecting the shape of the BDT distributions are considered with Gaussian probability distributions. The BDT distributions from the five jet categories and from the three data-taking periods (the 2016 ones being taken from the analysis described in ref. [24]) are used to derive the final results. Table 1 shows the number of events per process and jet category, derived from the simultaneous fit for the $2017+2018$ data and simulation.

The expected and observed exclusion limits on the product of the cross section and branching fraction for the two couplings are shown in figure 5 separately for each jet category as well as their combination. The b3j4 category has the highest sensitivity to both couplings since it corresponds to the canonical jet content of the dominant TT signal contribution, while the $\mathrm{SM} t \overline{\mathrm{t}}$ contribution is suppressed due to the requirement of a third $\mathrm{b}$ jet. The $\mathrm{b} 2 \mathrm{j} 3$ and $\mathrm{b} 4 \mathrm{j} 4$ categories show reversed sensitivity between Hut and Hct couplings due to the much higher $\mathrm{b}$ jet misidentification probability of $\mathrm{c}$ jets compared to $\mathrm{u}$ jets.

The exclusion limit on the cross section can be interpreted in terms of an exclusion on the anomalous coupling $\kappa_{\mathrm{Hqt}}$ since the signal cross section scales linearly with $\kappa_{\mathrm{Hqt}}^{2}$. Using the signal cross sections for $\kappa_{\text {Hqt }}=1$ from section 3 leads to observed (expected) limits of $\kappa_{\text {Hut }}<0.074(0.087)$ and $\kappa_{\text {Hct }}<0.081(0.078)$ at $95 \%$ CL. This can in turn be interpreted in terms of a limit on the $\mathrm{t} \rightarrow \mathrm{Hq}$ branching fraction thanks to the relation

$$
\kappa_{\mathrm{Hqt}}^{2}=\mathcal{B}(\mathrm{t} \rightarrow \mathrm{Hq}) \frac{\Gamma_{\mathrm{t}}}{\Gamma_{\mathrm{Hqt}}},
$$



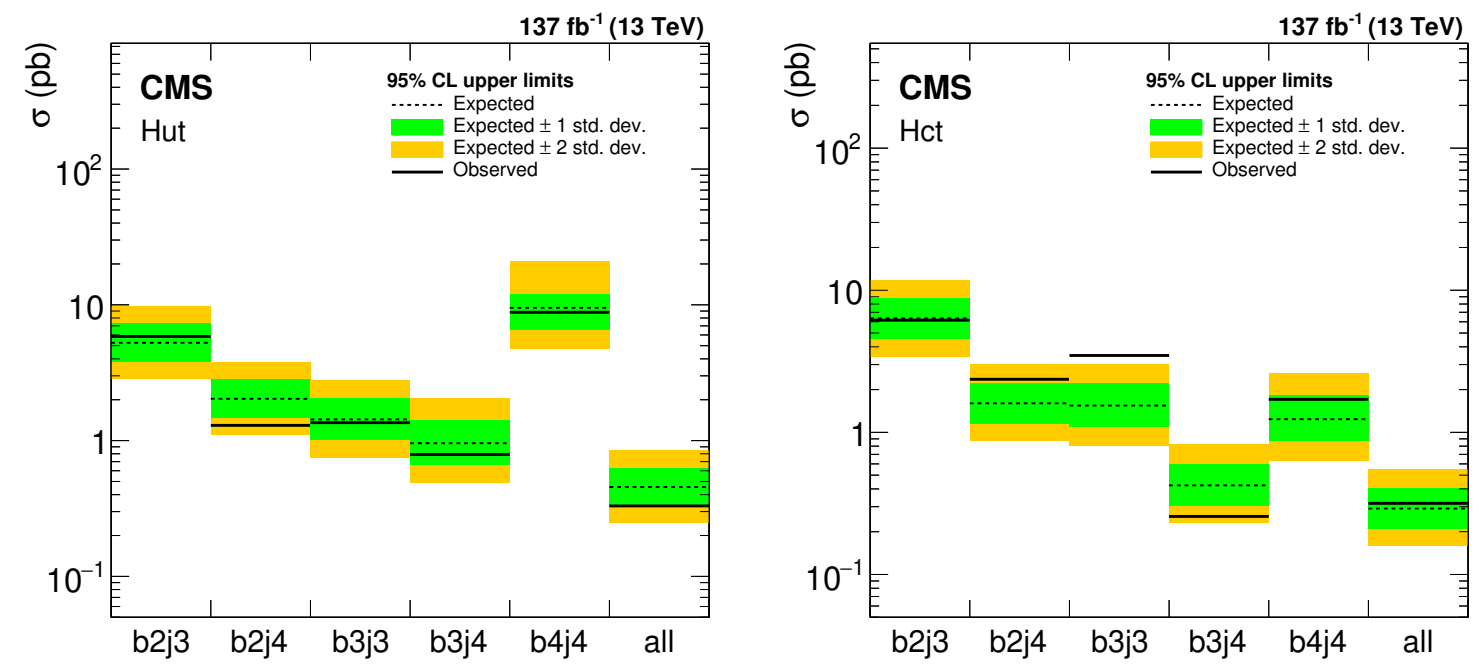

Figure 5. Excluded limits on the product of the cross section and branching fraction at 95\% CL for the Hut (left) and Hct (right) couplings obtained using the BDT distributions. Each jet category and their combination are shown separately.
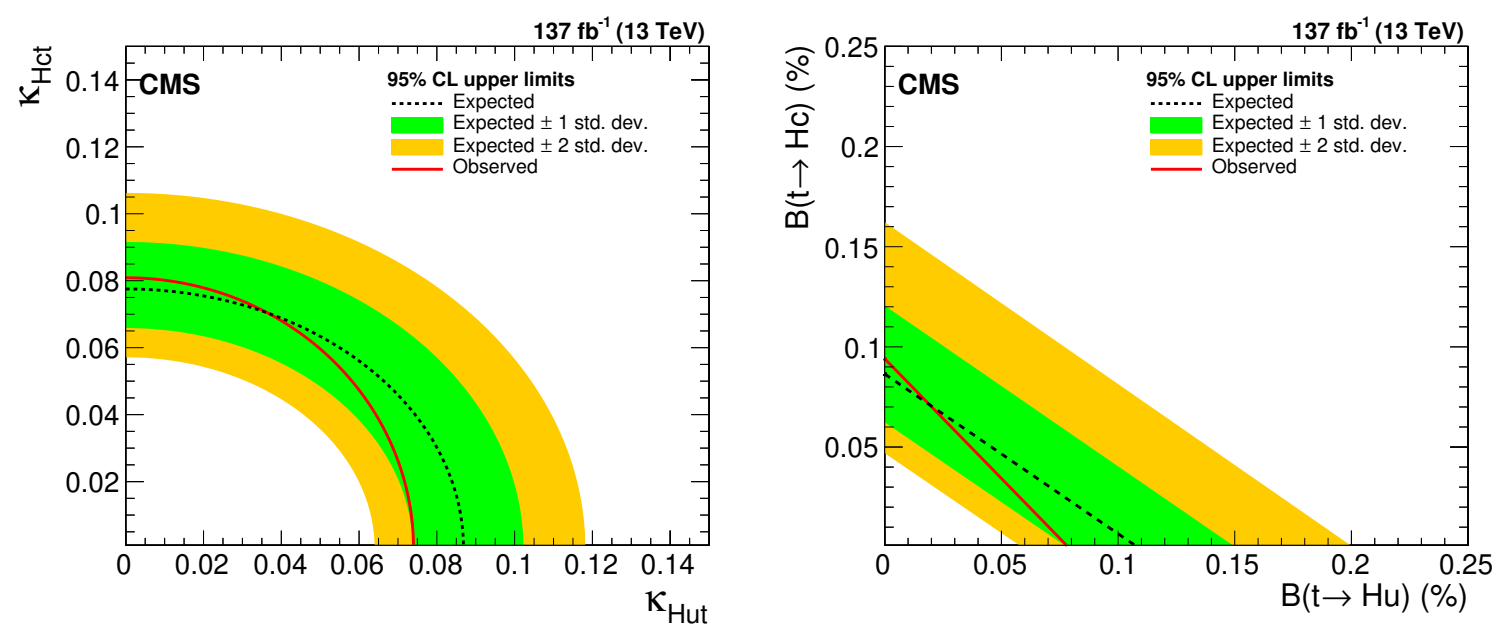

Figure 6. Upper limits on the couplings $\kappa_{\mathrm{Hut}}$ and $\kappa_{\mathrm{Hct}}$ (left), and the branching fractions $\mathcal{B}(\mathrm{t} \rightarrow$ $\mathrm{Hu})$ and $\mathcal{B}(\mathrm{t} \rightarrow \mathrm{Hc})$ (right) at $95 \% \mathrm{CL}$.

with $\Gamma_{\mathrm{t}}=1.32 \mathrm{GeV}$ at NNLO [73] and $\Gamma_{\mathrm{Hut}}^{\kappa_{\mathrm{Hut}}=1}=\Gamma_{\mathrm{Hct}}^{\kappa_{\mathrm{Hct}}=1}=0.19 \mathrm{GeV}$. The observed (expected) $95 \%$ CL exclusion limits on the branching fractions are $\mathcal{B}(\mathrm{t} \rightarrow \mathrm{Hu})<0.079(0.11) \%$ and $\mathcal{B}(\mathrm{t} \rightarrow \mathrm{Hc})<0.094(0.086) \%$. The limits for a single nonvanishing coupling are interpolated by assuming a linear relationship between the branching fractions. The resulting two-dimensional limits for the branching fractions and couplings are shown in figure 6 . The results presented here improve by a factor of 3-6 compared to those obtained in the same decay channel with the 2016 data set [24], and are comparable to the observed limits from the $13 \mathrm{TeV}$ analysis in the diphoton channel [25]. 


\section{Summary}

A search for flavor-changing neutral current interactions in events with a top quark $(\mathrm{t})$ decaying leptonically and a Higgs boson $(\mathrm{H})$ decaying to a bottom quark-antiquark pair has been presented. The search uses the LHC data, collected at $\sqrt{s}=13 \mathrm{TeV}$ in $2016-2018$ and corresponding to an integrated luminosity of $137 \mathrm{fb}^{-1}$ of proton-proton collisions. Events are analyzed, in the single-lepton channel containing a muon or electron in addition to the presence of at least three jets, where at least two of them are identified as originating from the hadronization of a bottom quark. No significant deviation from the standard model prediction has been observed and upper limits on the branching fractions $\mathcal{B}(\mathrm{t} \rightarrow \mathrm{Hq})$ have been set where q refers to the up $(\mathrm{u})$ and charm quarks (c); their observed (expected) excluded values at $95 \%$ confidence level are $\mathcal{B}(\mathrm{t} \rightarrow \mathrm{Hu})<0.079(0.11) \%$ and $\mathcal{B}(\mathrm{t} \rightarrow \mathrm{Hc})<$ $0.094(0.086) \%$. The observed limits reach the order of $10^{-4}$ in terms of branching fraction compared to the prediction from several well-known extensions of standard model that predict the values as high as $10^{-5}$ to $10^{-3}$. This search substantially improves upon previous CMS results in the same final state by exploiting the larger integrated luminosity of $137 \mathrm{fb}^{-1}$ and by using advanced multivariate analysis techniques to perform the kinematic event reconstruction and signal extraction.

\section{Acknowledgments}

We congratulate our colleagues in the CERN accelerator departments for the excellent performance of the LHC and thank the technical and administrative staffs at CERN and at other CMS institutes for their contributions to the success of the CMS effort. In addition, we gratefully acknowledge the computing centres and personnel of the Worldwide LHC Computing Grid and other centres for delivering so effectively the computing infrastructure essential to our analyses. Finally, we acknowledge the enduring support for the construction and operation of the LHC, the CMS detector, and the supporting computing infrastructure provided by the following funding agencies: BMBWF and FWF (Austria); FNRS and FWO (Belgium); CNPq, CAPES, FAPERJ, FAPERGS, and FAPESP (Brazil); MES and BNSF (Bulgaria); CERN; CAS, MoST, and NSFC (China); MINCIENCIAS (Colombia); MSES and CSF (Croatia); RIF (Cyprus); SENESCYT (Ecuador); MoER, ERC PUT and ERDF (Estonia); Academy of Finland, MEC, and HIP (Finland); CEA and CNRS/IN2P3 (France); BMBF, DFG, and HGF (Germany); GSRI (Greece); NKFIA (Hungary); DAE and DST (India); IPM (Iran); SFI (Ireland); INFN (Italy); MSIP and NRF (Republic of Korea); MES (Latvia); LAS (Lithuania); MOE and UM (Malaysia); BUAP, CINVESTAV, CONACYT, LNS, SEP, and UASLP-FAI (Mexico); MOS (Montenegro); MBIE (New Zealand); PAEC (Pakistan); MSHE and NSC (Poland); FCT (Portugal); JINR (Dubna); MON, RosAtom, RAS, RFBR, and NRC KI (Russia); MESTD (Serbia); MCIN/AEI and PCTI (Spain); MOSTR (Sri Lanka); Swiss Funding Agencies (Switzerland); MST (Taipei); ThEPCenter, IPST, STAR, and NSTDA (Thailand); TUBITAK and TAEK (Turkey); NASU (Ukraine); STFC (United Kingdom); DOE and NSF (U.S.A.).

Individuals have received support from the Marie-Curie program and the European Research Council and Horizon 2020 Grant, contract Nos. 675440, 724704, 752730, 758316, 
765710, 824093, 884104, and COST Action CA16108 (European Union); the Leventis Foundation; the Alfred P. Sloan Foundation; the Alexander von Humboldt Foundation; the Belgian Federal Science Policy Office; the Fonds pour la Formation à la Recherche dans l'Industrie et dans l'Agriculture (FRIA-Belgium); the Agentschap voor Innovatie door Wetenschap en Technologie (IWT-Belgium); the F.R.S.-FNRS and FWO (Belgium) under the "Excellence of Science - EOS" — be.h project n. 30820817; the Beijing Municipal Science \& Technology Commission, No. Z191100007219010; the Ministry of Education, Youth and Sports (MEYS) of the Czech Republic; the Deutsche Forschungsgemeinschaft (DFG), under Germany's Excellence Strategy - EXC 2121 "Quantum Universe" - 390833306, and under project number 400140256 - GRK2497; the Lendület ("Momentum") Program and the János Bolyai Research Scholarship of the Hungarian Academy of Sciences, the New National Excellence Program ÚNKP, the NKFIA research grants 123842, 123959, 124845, 124850, 125105, 128713, 128786, and 129058 (Hungary); the Council of Science and Industrial Research, India; the Latvian Council of Science; the Ministry of Science and Higher Education and the National Science Center, contracts Opus 2014/15/B/ST2/03998 and 2015/19/B/ST2/02861 (Poland); the Fundação para a Ciência e a Tecnologia, grant CEECIND/01334/2018 (Portugal); the National Priorities Research Program by Qatar National Research Fund; the Ministry of Science and Higher Education, projects no. 14.W03.31.0026 and no. FSWW-2020-0008, and the Russian Foundation for Basic Research, project No.19-42-703014 (Russia); MCIN/AEI/10.13039/501100011033, ERDF "a way of making Europe", and the Programa Estatal de Fomento de la Investigación Científica y Técnica de Excelencia María de Maeztu, grant MDM-2017-0765 and Programa Severo Ochoa del Principado de Asturias (Spain); the Stavros Niarchos Foundation (Greece); the Rachadapisek Sompot Fund for Postdoctoral Fellowship, Chulalongkorn University and the Chulalongkorn Academic into Its 2nd Century Project Advancement Project (Thailand); the Kavli Foundation; the Nvidia Corporation; the SuperMicro Corporation; the Welch Foundation, contract C-1845; and the Weston Havens Foundation (U.S.A.).

Open Access. This article is distributed under the terms of the Creative Commons Attribution License (CC-BY 4.0), which permits any use, distribution and reproduction in any medium, provided the original author(s) and source are credited.

\section{References}

[1] ATLAS collaboration, Observation of a new particle in the search for the Standard Model Higgs boson with the ATLAS detector at the LHC, Phys. Lett. B 716 (2012) 1 [arXiv: 1207.7214] [INSPIRE].

[2] CMS collaboration, Observation of a new boson at a mass of $125 \mathrm{GeV}$ with the CMS experiment at the LHC, Phys. Lett. B 716 (2012) 30 [arXiv:1207.7235] [INSPIRE].

[3] CMS collaboration, Observation of a new boson with mass near $125 \mathrm{GeV}$ in pp collisions at $\sqrt{s}=7$ and $8 \mathrm{TeV}$, JHEP 06 (2013) 081 [arXiv:1303.4571] [INSPIRE].

[4] ATLAS and CMS collaborations, Measurements of the Higgs boson production and decay rates and constraints on its couplings from a combined ATLAS and CMS analysis of the LHC pp collision data at $\sqrt{s}=7$ and $8 \mathrm{TeV}$, JHEP 08 (2016) 045 [arXiv:1606.02266] [INSPIRE]. 
[5] CMS collaboration, Measurement of the top quark Yukawa coupling from t $\bar{t}$ kinematic distributions in the dilepton final state in proton-proton collisions at $\sqrt{s}=13 \mathrm{TeV}$, Phys. Rev. D 102 (2020) 092013 [arXiv: 2009.07123] [INSPIRE].

[6] B.A. Dobrescu and C.T. Hill, Electroweak symmetry breaking via top condensation seesaw, Phys. Rev. Lett. 81 (1998) 2634 [hep-ph/9712319] [inSPIRE].

[7] R.S. Chivukula, B.A. Dobrescu, H. Georgi and C.T. Hill, Top quark seesaw theory of electroweak symmetry breaking, Phys. Rev. D 59 (1999) 075003 [hep-ph/9809470] [INSPIRE].

[8] D. Delepine, J.M. Gerard and R. Gonzalez Felipe, Is the standard Higgs scalar elementary?, Phys. Lett. B 372 (1996) 271 [hep-ph/9512339] [INSPIRE].

[9] S.L. Glashow, J. Iliopoulos and L. Maiani, Weak interactions with lepton-hadron symmetry, Phys. Rev. D 2 (1970) 1285 [INSPIRE].

[10] J.A. Aguilar-Saavedra, Top flavor-changing neutral interactions: theoretical expectations and experimental detection, Acta Phys. Polon. B 35 (2004) 2695 [hep-ph/0409342] [INSPIRE].

[11] B. Mele, S. Petrarca and A. Soddu, A new evaluation of the $t \rightarrow c H$ decay width in the Standard Model, Phys. Lett. B 435 (1998) 401 [hep-ph/9805498] [INSPIRE].

[12] G. Eilam, J.L. Hewett and A. Soni, Rare decays of the top quark in the standard and two Higgs doublet models, Phys. Rev. D 44 (1991) 1473 [Erratum ibid. 59 (1999) 039901] [INSPIRE].

[13] J. Guasch and J. Solà, FCNC top quark decays: a door to SUSY physics in high luminosity colliders?, Nucl. Phys. B 562 (1999) 3 [hep-ph/9906268] [INSPIRE].

[14] G. Eilam, A. Gemintern, T. Han, J.M. Yang and X. Zhang, Top quark rare decay $t \rightarrow c h$ in R-parity violating SUSY, Phys. Lett. B 510 (2001) 227 [hep-ph/0102037] [INSPIRE].

[15] T.P. Cheng and M. Sher, Mass matrix ansatz and flavor nonconservation in models with multiple Higgs doublets, Phys. Rev. D 35 (1987) 3484 [inSPIRE].

[16] D. Atwood, L. Reina and A. Soni, Phenomenology of two Higgs doublet models with flavor changing neutral currents, Phys. Rev. D 55 (1997) 3156 [hep-ph/9609279] [INSPIRE].

[17] S. Bejar, J. Guasch and J. Solà, Loop induced flavor changing neutral decays of the top quark in a general two Higgs doublet model, Nucl. Phys. B 600 (2001) 21 [hep-ph/0011091] [INSPIRE].

[18] J.A. Aguilar-Saavedra, Effects of mixing with quark singlets, Phys. Rev. D 67 (2003) 035003 [Erratum ibid. 69 (2004) 099901] [hep-ph/0210112] [INSPIRE].

[19] A. Azatov, M. Toharia and L. Zhu, Higgs mediated FCNC's in warped extra dimensions, Phys. Rev. D 80 (2009) 035016 [arXiv:0906.1990] [INSPIRE].

[20] A. Crivellin, J. Heeck and P. Stoffer, A perturbed lepton-specific two-Higgs-doublet model facing experimental hints for physics beyond the Standard Model, Phys. Rev. Lett. 116 (2016) 081801 [arXiv: 1507.07567] [INSPIRE].

[21] ATLAS collaboration, Search for top quark decays $t \rightarrow q H$, with $H \rightarrow \gamma \gamma$, in $\sqrt{s}=13 \mathrm{TeV}$ pp collisions using the ATLAS detector, JHEP 10 (2017) 129 [arXiv:1707.01404] [INSPIRE].

[22] ATLAS collaboration, Search for flavor-changing neutral currents in top quark decays $t \rightarrow H c$ and $t \rightarrow H u$ in multilepton final states in proton-proton collisions at $\sqrt{s}=13 \mathrm{TeV}$ with the ATLAS detector, Phys. Rev. D 98 (2018) 032002 [arXiv:1805.03483] [INSPIRE]. 
[23] ATLAS collaboration, Search for top-quark decays $t \rightarrow H q$ with $36 \mathrm{fb}^{-1}$ of pp collision data at $\sqrt{s}=13 \mathrm{TeV}$ with the ATLAS detector, JHEP 05 (2019) 123 [arXiv:1812.11568] [INSPIRE].

[24] CMS collaboration, Search for the flavor-changing neutral current interactions of the top quark and the Higgs boson which decays into a pair of b quarks at $\sqrt{s}=13 \mathrm{TeV}$, JHEP 06 (2018) 102 [arXiv: 1712.02399] [INSPIRE].

[25] CMS collaboration, Search for flavor-changing neutral current interactions of the top quark and Higgs boson in final states with two photons in proton-proton collisions at $\sqrt{s}=13 \mathrm{TeV}$, submitted to Phys. Rev. Lett. [arXiv:2111.02219] [INSPIRE].

[26] HEPData record for this analysis, CMS-TOP-19-002, (2021).

[27] CMS collaboration, Performance of the CMS level-1 trigger in proton-proton collisions at $\sqrt{s}=13 \mathrm{TeV}, 2020 \mathrm{JINST} 15 \mathrm{P} 10017$ [arXiv:2006.10165] [INSPIRE].

[28] CMS collaboration, The CMS trigger system, 2017 JINST 12 P01020 [arXiv:1609.02366] [INSPIRE].

[29] CMS collaboration, The CMS experiment at the CERN LHC, 2008 JINST 3 S08004 [INSPIRE].

[30] J. Alwall et al., The automated computation of tree-level and next-to-leading order differential cross sections, and their matching to parton shower simulations, JHEP 07 (2014) 079 [arXiv: 1405.0301] [INSPIRE].

[31] A. Alloul, N.D. Christensen, C. Degrande, C. Duhr and B. Fuks, FeynRules $2.0-a$ complete toolbox for tree-level phenomenology, Comput. Phys. Commun. 185 (2014) 2250 [arXiv: 1310.1921] [INSPIRE].

[32] C. Degrande, C. Duhr, B. Fuks, D. Grellscheid, O. Mattelaer and T. Reiter, UFO - the Universal FeynRules Output, Comput. Phys. Commun. 183 (2012) 1201 [arXiv:1108.2040] [INSPIRE].

[33] J. Alwall et al., Comparative study of various algorithms for the merging of parton showers and matrix elements in hadronic collisions, Eur. Phys. J. C 53 (2008) 473 [arXiv:0706.2569] [INSPIRE].

[34] M. Czakon and A. Mitov, Top++: a program for the calculation of the top-pair cross-section at hadron colliders, Comput. Phys. Commun. 185 (2014) 2930 [arXiv:1112.5675] [INSPIRE].

[35] M. Czakon, D. Heymes, A. Mitov, D. Pagani, I. Tsinikos and M. Zaro, Top-pair production at the LHC through NNLO QCD and NLO EW, JHEP 10 (2017) 186 [arXiv:1705.04105] [INSPIRE].

[36] CMS collaboration, Measurement of differential t $\bar{t}$ production cross sections in the full kinematic range using lepton + jets events from proton-proton collisions at $\sqrt{s}=13 \mathrm{TeV}$, Phys. Rev. D 104 (2021) 092013 [arXiv:2108.02803] [INSPIRE].

[37] P. Nason, A new method for combining NLO QCD with shower Monte Carlo algorithms, JHEP 11 (2004) 040 [hep-ph/0409146] [INSPIRE].

[38] S. Frixione, P. Nason and C. Oleari, Matching NLO QCD computations with parton shower simulations: the POWHEG method, JHEP 11 (2007) 070 [arXiv:0709.2092] [INSPIRE]. 
[39] S. Alioli, P. Nason, C. Oleari and E. Re, A general framework for implementing NLO calculations in shower Monte Carlo programs: the POWHEG BOX, JHEP 06 (2010) 043 [arXiv: 1002.2581] [INSPIRE].

[40] CMS collaboration, Measurement of the cross section for $t \bar{t}$ production with additional jets and $b$ jets in pp collisions at $\sqrt{s}=13 \mathrm{TeV}$, JHEP 07 (2020) 125 [arXiv:2003.06467] [INSPIRE].

[41] E. Re, Single-top Wt-channel production matched with parton showers using the POWHEG method, Eur. Phys. J. C 71 (2011) 1547 [arXiv:1009.2450] [INSPIRE].

[42] M. Aliev, H. Lacker, U. Langenfeld, S. Moch, P. Uwer and M. Wiedermann, HATHOR: HAdronic Top and Heavy quarks crOss section calculatoR, Comput. Phys. Commun. 182 (2011) 1034 [arXiv: 1007.1327] [inSPIRE].

[43] P. Kant et al., HatHor for single top-quark production: updated predictions and uncertainty estimates for single top-quark production in hadronic collisions, Comput. Phys. Commun. 191 (2015) 74 [arXiv:1406.4403] [INSPIRE].

[44] N. Kidonakis, Two-loop soft anomalous dimensions for single top quark associated production with a $W^{-}$or $H^{-}$, Phys. Rev. D 82 (2010) 054018 [arXiv: 1005.4451] [INSPIRE].

[45] N. Kidonakis, NNLL threshold resummation for top-pair and single-top production, Phys. Part. Nucl. 45 (2014) 714 [arXiv:1210.7813] [INSPIRE].

[46] T. Sjöstrand et al., An introduction to PYTHIA 8.2, Comput. Phys. Commun. 191 (2015) 159 [arXiv: 1410.3012] [INSPIRE].

[47] NNPDF collaboration, Parton distributions for the LHC run II, JHEP 04 (2015) 040 [arXiv: 1410.8849] [INSPIRE].

[48] CMS collaboration, Extraction and validation of a new set of CMS PYTHIA8 tunes from underlying-event measurements, Eur. Phys. J. C 80 (2020) 4 [arXiv:1903.12179] [INSPIRE].

[49] CMS collaboration, Measurement of the inelastic proton-proton cross section at $\sqrt{s}=13 \mathrm{TeV}$, JHEP 07 (2018) 161 [arXiv:1802.02613] [INSPIRE].

[50] GEANT4 collaboration, GEANT4 - a simulation toolkit, Nucl. Instrum. Meth. A 506 (2003) 250 [INSPIRE].

[51] CMS collaboration, Particle-flow reconstruction and global event description with the CMS detector, 2017 JINST 12 P10003 [arXiv:1706.04965] [INSPIRE].

[52] CMS collaboration, Performance of missing transverse momentum reconstruction in proton-proton collisions at $\sqrt{s}=13 \mathrm{TeV}$ using the CMS detector, 2019 JINST $14 \mathrm{P} 07004$ [arXiv: 1903.06078] [INSPIRE].

[53] M. Cacciari, G.P. Salam and G. Soyez, The anti- $k_{t}$ jet clustering algorithm, JHEP 04 (2008) 063 [arXiv:0802.1189] [INSPIRE].

[54] M. Cacciari, G.P. Salam and G. Soyez, FastJet user manual, Eur. Phys. J. C 72 (2012) 1896 [arXiv:1111.6097] [INSPIRE].

[55] CMS collaboration, Determination of jet energy calibration and transverse momentum resolution in CMS, 2011 JINST 6 P11002 [arXiv:1107.4277] [INSPIRE].

[56] CMS collaboration, Identification and filtering of uncharacteristic noise in the CMS hadron calorimeter, 2010 JINST 5 T03014 [arXiv:0911.4881] [INSPIRE]. 
[57] CMS collaboration, Identification of heavy-flavour jets with the CMS detector in pp collisions at $13 \mathrm{TeV}, 2018$ JINST 13 P05011 [arXiv:1712.07158] [INSPIRE].

[58] CMS collaboration, Measurement of the inclusive $W$ and $Z$ production cross sections in $p p$ collisions at $\sqrt{s}=7 \mathrm{TeV}$, JHEP 10 (2011) 132 [arXiv:1107.4789] [INSPIRE].

[59] CMS collaboration, Performance of the CMS muon detector and muon reconstruction with proton-proton collisions at $\sqrt{s}=13 \mathrm{TeV}, 2018$ JINST $13 \mathrm{P} 06015$ [arXiv:1804.04528] [INSPIRE].

[60] CMS collaboration, Electron and photon reconstruction and identification with the CMS experiment at the CERN LHC, 2021 JINST 16 P05014 [arXiv:2012.06888] [INSPIRE].

[61] K. He, X. Zhang, S. Ren and J. Sun, Deep residual learning for image recognition, in 2016 IEEE conference on Computer Vision and Pattern Recognition (CVPR), IEEE, (2016), pg. 770 [arXiv: 1512.03385] [INSPIRE].

[62] M. Erdmann, B. Fischer and M. Rieger, Jet-parton assignment in $t \bar{t} H$ events using deep learning, 2017 JINST 12 P08020 [arXiv:1706.01117] [INSPIRE].

[63] CMS collaboration, CMS luminosity measurement for the 2017 data-taking period at $\sqrt{s}=13 \mathrm{TeV}$, Tech. Rep. CMS-PAS-LUM-17-004, CERN, Geneva, Switzerland (2018).

[64] CMS collaboration, CMS luminosity measurement for the 2018 data-taking period at $\sqrt{s}=13 \mathrm{TeV}$, Tech. Rep. CMS-PAS-LUM-18-002, CERN, Geneva, Switzerland (2019).

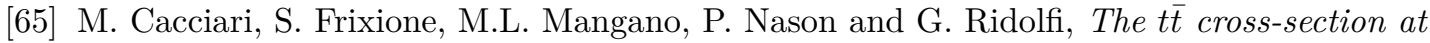
$1.8 \mathrm{TeV}$ and $1.96 \mathrm{TeV}$ : a study of the systematics due to parton densities and scale dependence, JHEP 04 (2004) 068 [hep-ph/0303085] [INSPIRE].

[66] S. Catani, D. de Florian, M. Grazzini and P. Nason, Soft gluon resummation for Higgs boson production at hadron colliders, JHEP 07 (2003) 028 [hep-ph/0306211] [INSPIRE].

[67] J. Butterworth et al., PDF4LHC recommendations for LHC run II, J. Phys. G 43 (2016) 023001 [arXiv: 1510.03865 ] [INSPIRE].

[68] R.J. Barlow and C. Beeston, Fitting using finite Monte Carlo samples, Comput. Phys. Commun. 77 (1993) 219 [INSPIRE].

[69] J.S. Conway, Incorporating nuisance parameters in likelihoods for multisource spectra, in PHYSTAT 2011, CERN-2011-006, CERN, Geneva, Switzerland (2011), pg. 115. [arXiv: 1103.0354] [INSPIRE].

[70] T. Junk, Confidence level computation for combining searches with small statistics, Nucl. Instrum. Meth. A 434 (1999) 435 [hep-ex/9902006] [INSPIRE].

[71] A.L. Read, Presentation of search results: the $C L_{s}$ technique, J. Phys. G 28 (2002) 2693 [INSPIRE].

[72] G. Cowan, K. Cranmer, E. Gross and O. Vitells, Asymptotic formulae for likelihood-based tests of new physics, Eur. Phys. J. C 71 (2011) 1554 [Erratum ibid. 73 (2013) 2501] [arXiv: 1007.1727] [INSPIRE].

[73] J. Gao, C.S. Li and H.X. Zhu, Top quark decay at next-to-next-to leading order in QCD, Phys. Rev. Lett. 110 (2013) 042001 [arXiv: 1210.2808] [INSPIRE]. 


\section{The CMS collaboration}

\section{Yerevan Physics Institute, Yerevan, Armenia}

A. Tumasyan

\section{Institut für Hochenergiephysik, Vienna, Austria}

W. Adam (D), J.W. Andrejkovic, T. Bergauer (D), S. Chatterjee (D), K. Damanakis, M. Dragicevic (D), A. Escalante Del Valle (D), R. Frühwirth ${ }^{1}$, M. Jeitler ${ }^{1}$ (D), N. Krammer, L. Lechner (D), D. Liko, I. Mikulec, P. Paulitsch, F.M. Pitters, J. Schieck ${ }^{1}$ (D), R. Schöfbeck (D), D. Schwarz, S. Templ (D), W. Waltenberger (D), C.-E. Wulz ${ }^{1}$ (D)

Institute for Nuclear Problems, Minsk, Belarus

V. Chekhovsky, A. Litomin, V. Makarenko

\section{Universiteit Antwerpen, Antwerpen, Belgium}

M.R. Darwish ${ }^{2}$, E.A. De Wolf, T. Janssen (D), T. Kello ${ }^{3}$, A. Lelek (D), H. Rejeb Sfar, P. Van Mechelen (D), S. Van Putte, N. Van Remortel (D)

\section{Vrije Universiteit Brussel, Brussel, Belgium}

F. Blekman (D), E.S. Bols (D), J. D'Hondt (D), M. Delcourt, H. El Faham (D), S. Lowette (D), S. Moortgat (D), A. Morton (D), D. Müller (D), A.R. Sahasransu (D), S. Tavernier (D), W. Van Doninck

\section{Université Libre de Bruxelles, Bruxelles, Belgium}

D. Beghin, B. Bilin (D), B. Clerbaux (D), G. De Lentdecker, L. Favart (D), A. Grebenyuk, A.K. Kalsi (D), K. Lee, M. Mahdavikhorrami, I. Makarenko (D), L. Moureaux (D), L. Pétré, A. Popov (D), N. Postiau, E. Starling (D), L. Thomas (D), M. Vanden Bemden, C. Vander Velde (iD, P. Vanlaer (iD

\section{Ghent University, Ghent, Belgium}

T. Cornelis (D), D. Dobur, J. Knolle (D), L. Lambrecht, G. Mestdach, M. Niedziela (D), C. Roskas, A. Samalan, K. Skovpen (ID), M. Tytgat (D), B. Vermassen, L. Wezenbeek

\section{Université Catholique de Louvain, Louvain-la-Neuve, Belgium}

A. Benecke, A. Bethani (D), G. Bruno, F. Bury (D), C. Caputo (D), P. David (D), C. Delaere (D), I.S. Donertas (D), A. Giammanco (D), K. Jaffel, Sa. Jain (D), V. Lemaitre, K. Mondal (D), J. Prisciandaro, A. Taliercio, M. Teklishyn (D), T.T. Tran, P. Vischia (D), S. Wertz (D)

Centro Brasileiro de Pesquisas Fisicas, Rio de Janeiro, Brazil

G.A. Alves (D), C. Hensel, A. Moraes (D), P. Rebello Teles (D)

Universidade do Estado do Rio de Janeiro, Rio de Janeiro, Brazil

W.L. Aldá Júnior (D), M. Alves Gallo Pereira (D), M. Barroso Ferreira Filho, H. Brandao Malbouisson, W. Carvalho (D), J. Chinellato ${ }^{4}$, E.M. Da Costa (D), G.G. Da Silveira ${ }^{5}$ (D), D. De Jesus Damiao (D), S. Fonseca De Souza (D), C. Mora Herrera (D), K. Mota Amarilo, L. Mundim (D), H. Nogima, A. Santoro, S.M. Silva Do Amaral (D), A. Sznajder (D), M. Thiel, F. Torres Da Silva De Araujo ${ }^{6}$ (D) A. Vilela Pereira (D) 
Universidade Estadual Paulista (a), Universidade Federal do ABC (b), São Paulo, Brazil

C.A. Bernardes ${ }^{5}$ (D), L. Calligaris (D), T.R. Fernandez Perez Tomei (D), E.M. Gregores (D), D.S. Lemos (1D, P.G. Mercadante (D), S.F. Novaes (D), Sandra S. Padula (D)

Institute for Nuclear Research and Nuclear Energy, Bulgarian Academy of Sciences, Sofia, Bulgaria

A. Aleksandrov, G. Antchev (D), R. Hadjiiska, P. Iaydjiev, M. Misheva, M. Rodozov, M. Shopova, G. Sultanov

University of Sofia, Sofia, Bulgaria

A. Dimitrov, T. Ivanov, L. Litov (D), B. Pavlov, P. Petkov, A. Petrov

Beihang University, Beijing, China

T. Cheng (D), T. Javaid ${ }^{7}$, M. Mittal, L. Yuan

Department of Physics, Tsinghua University, Beijing, China

M. Ahmad (D), G. Bauer, C. $\operatorname{Dozen}^{8}$ (D), Z. Hu (D), J. Martins ${ }^{9}$ (D), Y. Wang, K. Yi ${ }^{10,11}$

Institute of High Energy Physics, Beijing, China

E. Chapon (D), G.M. Chen ${ }^{7}$ (D) H.S. Chen ${ }^{7}$ (D), M. Chen (D), F. Iemmi, A. Kapoor (D),

D. Leggat, H. Liao, Z.-A. Liu ${ }^{7}$ (D) V. Milosevic (D), F. Monti (D), R. Sharma (D), J. Tao (D),

J. Thomas-Wilsker, J. Wang (D), H. Zhang (D), J. Zhao (D)

State Key Laboratory of Nuclear Physics and Technology, Peking University, Beijing, China

A. Agapitos, Y. An, Y. Ban, C. Chen, A. Levin (D), Q. Li (D), X. Lyu, Y. Mao, S.J. Qian,

D. Wang (iD, J. Xiao

Sun Yat-Sen University, Guangzhou, China

M. Lu, Z. You (D)

Institute of Modern Physics and Key Laboratory of Nuclear Physics and Ionbeam Application (MOE) - Fudan University, Shanghai, China

X. $\mathrm{Gao}^{3}$, H. Okawa (D), Y. Zhang (D)

Zhejiang University, Hangzhou, China, Zhejiang, China

Z. Lin (D), M. Xiao (D)

Universidad de Los Andes, Bogota, Colombia

C. Avila (D), A. Cabrera (D), C. Florez (D), J. Fraga

Universidad de Antioquia, Medellin, Colombia

J. Mejia Guisao, F. Ramirez, J.D. Ruiz Alvarez (D), C.A. Salazar González (D)

University of Split, Faculty of Electrical Engineering, Mechanical Engineering and Naval Architecture, Split, Croatia

D. Giljanovic, N. Godinovic (D), D. Lelas (D), I. Puljak (D) 
University of Split, Faculty of Science, Split, Croatia

Z. Antunovic, M. Kovac, T. Sculac (iD)

Institute Rudjer Boskovic, Zagreb, Croatia

V. Brigljevic (D), D. Ferencek (D), D. Majumder (D), M. Roguljic, A. Starodumov ${ }^{12}$ (D),

T. Susa (iD)

University of Cyprus, Nicosia, Cyprus

A. Attikis (D), K. Christoforou, E. Erodotou, A. Ioannou, G. Kole (D), M. Kolosova, S. Konstantinou, J. Mousa (D), C. Nicolaou, F. Ptochos (D), P.A. Razis, H. Rykaczewski, H. Saka (D)

Charles University, Prague, Czech Republic

M. Finger ${ }^{13}$, M. Finger Jr. ${ }^{13}$ (D), A. Kveton

Escuela Politecnica Nacional, Quito, Ecuador

E. Ayala

Universidad San Francisco de Quito, Quito, Ecuador

E. Carrera Jarrin (D)

Academy of Scientific Research and Technology of the Arab Republic of Egypt, Egyptian Network of High Energy Physics, Cairo, Egypt

A. Ellithi Kamel ${ }^{14}$, S. Khalil ${ }^{15}$

Center for High Energy Physics (CHEP-FU), Fayoum University, El-Fayoum, Egypt

A. Lotfy (D), M.A. Mahmoud (D)

National Institute of Chemical Physics and Biophysics, Tallinn, Estonia

S. Bhowmik (D), R.K. Dewanjee (D), K. Ehataht, M. Kadastik, S. Nandan, C. Nielsen, J. Pata, M. Raidal(D, L. Tani, C. Veelken

Department of Physics, University of Helsinki, Helsinki, Finland

P. Eerola (D), L. Forthomme (D), H. Kirschenmann (D), K. Osterberg (D), M. Voutilainen (iD

Helsinki Institute of Physics, Helsinki, Finland

S. Bharthuar, E. Brücken (D), F. Garcia (D), J. Havukainen (D), M.S. Kim (D), R. Kinnunen, T. Lampén, K. Lassila-Perini (D), S. Lehti (D), T. Lindén, M. Lotti, L. Martikainen, M. Myllymäki, J. Ott (D), H. Siikonen, E. Tuominen (D), J. Tuominiemi

Lappeenranta University of Technology, Lappeenranta, Finland

P. Luukka (D), H. Petrow, T. Tuuva

IRFU, CEA, Université Paris-Saclay, Gif-sur-Yvette, France

C. Amendola (iD, M. Besancon, F. Couderc (D), M. Dejardin, D. Denegri, J.L. Faure,

F. Ferri (D), S. Ganjour, P. Gras, G. Hamel de Monchenault (D), P. Jarry, B. Lenzi (D),

E. Locci, J. Malcles, J. Rander, A. Rosowsky (D), M.Ö. Sahin (D), A. Savoy-Navarro ${ }^{16}$,

M. Titov (D), G.B. Yu (D) 
Laboratoire Leprince-Ringuet, CNRS/IN2P3, Ecole Polytechnique, Institut Polytechnique de Paris, Palaiseau, France

S. Ahuja (D), F. Beaudette (D), M. Bonanomi (D), A. Buchot Perraguin, P. Busson, A. Cappati, C. Charlot, O. Davignon, B. Diab, G. Falmagne (D), S. Ghosh, R. Granier de Cassagnac (D), A. Hakimi, I. Kucher (D), J. Motta, M. Nguyen (D), C. Ochando (D), P. Paganini (D), J. Rembser, R. Salerno (D), U. Sarkar (D), J.B. Sauvan (D), Y. Sirois (D), A. Tarabini, A. Zabi, A. Zghiche (D)

Université de Strasbourg, CNRS, IPHC UMR 7178, Strasbourg, France J.-L. Agram ${ }^{17}$ (D), J. Andrea, D. Apparu, D. Bloch (D), G. Bourgatte, J.-M. Brom, E.C. Chabert, C. Collard (D, D. Darej, J.-C. Fontaine ${ }^{17}$, U. Goerlach, C. Grimault, A.C. Le Bihan, E. Nibigira (D), P. Van Hove (D)

Institut de Physique des 2 Infinis de Lyon (IP2I ), Villeurbanne, France

E. Asilar (ID, S. Beauceron (ID, C. Bernet (D), G. Boudoul, C. Camen, A. Carle, N. Chanon (D), D. Contardo, P. Depasse (D), H. El Mamouni, J. Fay, S. Gascon (D), M. Gouzevitch (D), B. Ille, I.B. Laktineh, H. Lattaud (D), A. Lesauvage (D), M. Lethuillier (D), L. Mirabito, S. Perries, K. Shchablo, V. Sordini (D), L. Torterotot (D), G. Touquet, M. Vander Donckt, S. Viret

\section{Georgian Technical University, Tbilisi, Georgia}

A. Khvedelidze ${ }^{13}$ (D) I. Lomidze, Z. Tsamalaidze ${ }^{13}$

\section{RWTH Aachen University, I. Physikalisches Institut, Aachen, Germany}

V. Botta, L. Feld (D), K. Klein, M. Lipinski, D. Meuser, A. Pauls, N. Röwert, J. Schulz, M. Teroerde

RWTH Aachen University, III. Physikalisches Institut A, Aachen, Germany

A. Dodonova, D. Eliseev, M. Erdmann (D), P. Fackeldey (D), B. Fischer, S. Ghosh (D), T. Hebbeker (D), K. Hoepfner, F. Ivone, L. Mastrolorenzo, M. Merschmeyer (D), A. Meyer (D), G. Mocellin, S. Mondal, S. Mukherjee (D), D. Noll (D), A. Novak, T. Pook (D, A. Pozdnyakov (D), Y. Rath, H. Reithler, J. Roemer, A. Schmidt (D), S.C. Schuler, A. Sharma (D), L. Vigilante, S. Wiedenbeck, S. Zaleski

RWTH Aachen University, III. Physikalisches Institut B, Aachen, Germany C. Dziwok, G. Flügge, W. Haj Ahmad ${ }^{18}$ (D), O. Hlushchenko, T. Kress, A. Nowack (D), C. Pistone, O. Pooth, D. Roy (D), A. Stahl ${ }^{19}$ (D), T. Ziemons (D), A. Zotz

\section{Deutsches Elektronen-Synchrotron, Hamburg, Germany}

H. Aarup Petersen, M. Aldaya Martin, P. Asmuss, S. Baxter, M. Bayatmakou, O. Behnke, A. Bermúdez Martínez, S. Bhattacharya, A.A. Bin Anuar (D), K. Borras ${ }^{20}$, D. Brunner, A. Campbell (D), A. Cardini (D), C. Cheng, F. Colombina, S. Consuegra Rodríguez (D), G. Correia Silva, V. Danilov, M. De Silva, L. Didukh, G. Eckerlin, D. Eckstein, L.I. Estevez Banos (D), O. Filatov (D), E. Gallo ${ }^{21}$, A. Geiser, A. Giraldi, A. Grohsjean (D), M. Guthoff, A. Jafari ${ }^{22}$ (D), N.Z. Jomhari (D), H. Jung (D), A. Kasem ${ }^{20}$ (D) M. Kasemann (D), H. Kaveh (D), C. Kleinwort (D), R. Kogler (D), D. Krücker (D), W. Lange, J. Lidrych (D), K. Lipka, W. Lohmann ${ }^{23}$, R. Mankel, I.-A. Melzer-Pellmann (D), M. Mendizabal Morentin, J. Metwally, A.B. Meyer (D), M. Meyer (D), J. Mnich (D), A. Mussgiller, Y. Otarid, 
D. Pérez Adán (iD, D. Pitzl, A. Raspereza, B. Ribeiro Lopes, J. Rübenach, A. Saggio (D), A. Saibel (D), M. Savitskyi (D), M. Scham ${ }^{24}$, V. Scheurer, S. Schnake, P. Schütze, C. Schwanenberger ${ }^{21}$ (D) M. Shchedrolosiev, R.E. Sosa Ricardo (D), D. Stafford, N. Tonon (D), M. Van De Klundert (D), R. Walsh (D), D. Walter, Q. Wang (D), Y. Wen (D), K. Wichmann, L. Wiens, C. Wissing, S. Wuchterl (iD

\section{University of Hamburg, Hamburg, Germany}

R. Aggleton, S. Albrecht (D), S. Bein (D), L. Benato (D), P. Connor (D), K. De Leo (D), M. Eich, F. Feindt, A. Fröhlich, C. Garbers (D), E. Garutti (D), P. Gunnellini, M. Hajheidari, J. Haller (D), A. Hinzmann (D), G. Kasieczka, R. Klanner (D), T. Kramer, V. Kutzner, J. Lange (D), T. Lange (D), A. Lobanov (D), A. Malara (D), A. Nigamova, K.J. Pena Rodriguez, M. Rieger (D), O. Rieger, P. Schleper, M. Schröder (D), J. Schwandt (D), J. Sonneveld (D), H. Stadie, G. Steinbrück, A. Tews, I. Zoi (D)

\section{Karlsruher Institut fuer Technologie, Karlsruhe, Germany}

J. Bechtel (D), S. Brommer, M. Burkart, E. Butz (D), R. Caspart (D), T. Chwalek, W. De Boer ${ }^{\dagger}$, A. Dierlamm, A. Droll, K. El Morabit, N. Faltermann (D), M. Giffels, J.o. Gosewisch, A. Gottmann, F. Hartmann ${ }^{19}$ (D), C. Heidecker, U. Husemann (D, P. Keicher, R. Koppenhöfer, S. Maier, M. Metzler, S. Mitra (D), Th. Müller, M. Neukum, A. Nürnberg, G. Quast (D), K. Rabbertz (D), J. Rauser, D. Savoiu (D), M. Schnepf, D. Seith, I. Shvetsov, H.J. Simonis, R. Ulrich (ID, J. Van Der Linden, R.F. Von Cube, M. Wassmer, M. Weber (D), S. Wieland, R. Wolf (iD, S. Wozniewski, S. Wunsch

Institute of Nuclear and Particle Physics (INPP), NCSR Demokritos, Aghia Paraskevi, Greece

G. Anagnostou, G. Daskalakis, T. Geralis (D), A. Kyriakis, D. Loukas, A. Stakia (D)

\section{National and Kapodistrian University of Athens, Athens, Greece}

M. Diamantopoulou, D. Karasavvas, G. Karathanasis, P. Kontaxakis (D, C.K. Koraka, A. Manousakis-Katsikakis, A. Panagiotou, I. Papavergou, N. Saoulidou (D, K. Theofilatos (D), E. Tziaferi (D), K. Vellidis, E. Vourliotis

National Technical University of Athens, Athens, Greece

G. Bakas, K. Kousouris (D, I. Papakrivopoulos, G. Tsipolitis, A. Zacharopoulou

University of Ioánnina, Ioánnina, Greece

K. Adamidis, I. Bestintzanos, I. Evangelou (D), C. Foudas, P. Gianneios, P. Katsoulis, P. Kokkas, N. Manthos, I. Papadopoulos (D), J. Strologas (iD

MTA-ELTE Lendület CMS Particle and Nuclear Physics Group, Eötvös Loránd University, Budapest, Hungary

M. Csanad (D), K. Farkas, M.M.A. Gadallah ${ }^{25}$ (D), S. Lökös ${ }^{26}$ (D), P. Major, K. Mandal (D), A. Mehta (D), G. Pasztor (D), A.J. Rádl, O. Surányi, G.I. Veres (1D

Wigner Research Centre for Physics, Budapest, Hungary

M. Bartók ${ }^{27}$ (D), G. Bencze, C. Hajdu (D), D. Horvath ${ }^{28}$ (D), F. Sikler (D), V. Veszpremi (D) 
Institute of Nuclear Research ATOMKI, Debrecen, Hungary

S. Czellar, D. Fasanella (D), J. Karancsi ${ }^{27}$ (D), J. Molnar, Z. Szillasi, D. Teyssier

Institute of Physics, University of Debrecen, Debrecen, Hungary

P. Raics, Z.L. Trocsanyi ${ }^{29}$ (D), B. Ujvari

Karoly Robert Campus, MATE Institute of Technology, Gyongyos, Hungary

T. Csorgo ${ }^{30}$ (D) F. Nemes ${ }^{30}$, T. Novak

Indian Institute of Science (IISc), Bangalore, India

S. Choudhury, J.R. Komaragiri (D), D. Kumar, L. Panwar (D), P.C. Tiwari (D)

National Institute of Science Education and Research, HBNI, Bhubaneswar, India

S. Bahinipati ${ }^{31}$ (D), C. Kar (D), P. Mal, T. Mishra (D, V.K. Muraleedharan Nair Bindhu ${ }^{32}$, A. Nayak $^{32}$ (D), P. Saha, N. Sur (D), S.K. Swain, D. Vats ${ }^{32}$

Panjab University, Chandigarh, India

S. Bansal (D), S.B. Beri, V. Bhatnagar (D), G. Chaudhary (D), S. Chauhan (D), N. Dhingra ${ }^{33}$ (D),

R. Gupta, A. Kaur, M. Kaur (D), S. Kaur, P. Kumari (D), M. Meena, K. Sandeep (D), J.B. Singh (D), A.K. Virdi (iD)

University of Delhi, Delhi, India

A. Ahmed, A. Bhardwaj (D), B.C. Choudhary (D), M. Gola, S. Keshri (D), A. Kumar (D), M. Naimuddin (D), P. Priyanka (D), K. Ranjan, A. Shah (iD

Saha Institute of Nuclear Physics, HBNI, Kolkata, India

M. Bharti ${ }^{34}$, R. Bhattacharya, S. Bhattacharya (D), D. Bhowmik, S. Dutta, S. Dutta,

B. Gomber ${ }^{35}$ (D), M. Maity ${ }^{36}$, P. Palit (D), P.K. Rout (D), G. Saha, B. Sahu (D), S. Sarkar, M. Sharan, B. Singh ${ }^{34}$, S. Thakur ${ }^{34}$

Indian Institute of Technology Madras, Madras, India

P.K. Behera (D), S.C. Behera, P. Kalbhor (D), A. Muhammad, R. Pradhan, P.R. Pujahari, A. Sharma (D), A.K. Sikdar

Bhabha Atomic Research Centre, Mumbai, India

D. Dutta (D), V. Jha, V. Kumar (D), D.K. Mishra, K. Naskar ${ }^{37}$, P.K. Netrakanti, L.M. Pant, P. Shukla

Tata Institute of Fundamental Research-A, Mumbai, India

T. Aziz, S. Dugad, M. Kumar

Tata Institute of Fundamental Research-B, Mumbai, India

S. Banerjee (D), R. Chudasama, M. Guchait, S. Karmakar, S. Kumar, G. Majumder, K. Mazumdar, S. Mukherjee

Indian Institute of Science Education and Research (IISER), Pune, India K. Alpana, S. Dube (D), B. Kansal, A. Laha, S. Pandey (D), A. Rane(D), A. Rastogi (D), S. Sharma (iD 
Isfahan University of Technology, Isfahan, Iran

H. Bakhshiansohi ${ }^{38}$ (D), E. Khazaie, M. Zeinali ${ }^{39}$

Institute for Research in Fundamental Sciences (IPM), Tehran, Iran

S. Chenarani ${ }^{40}$, S.M. Etesami (D), M. Khakzad (D), M. Mohammadi Najafabadi (D)

University College Dublin, Dublin, Ireland

M. Grunewald (D)

INFN Sezione di Bari ${ }^{a}$, Bari, Italy, Università di Bari ${ }^{b}$, Bari, Italy, Politecnico di Bari ${ }^{c}$, Bari, Italy

M. Abbrescia $^{a, b}$ (D), R. Aly ${ }^{a, b, 41}$ (D), C. Aruta $^{a, b}$, A. Colaleo ${ }^{a}$ (D), D. Creanza ${ }^{a, c}$ (D), N. De Filippis $^{a, c} \mathbb{D}$, M. De Palma $^{a, b} \mathbb{D}$, A. Di Florio ${ }^{a, b}$, A. Di Pilato ${ }^{a, b}$ (D),

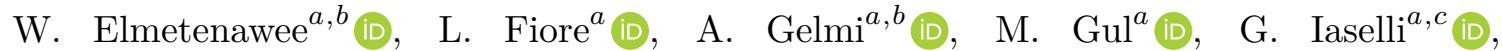
M. Ince $^{a, b}\left(\mathbb{D}, \quad\right.$ S. Lezki $^{a, b}$ (D), G. Maggi ${ }^{a, c}$ (D), M. Maggi ${ }^{a}$ (D), I. Margjeka ${ }^{a, b}$, V. Mastrapasqua ${ }^{a, b}$ (D), S. $\mathrm{My}^{a, b}$ (D), S. Nuzzo ${ }^{a, b}$ (D) A. Pellecchia ${ }^{a, b}$, A. Pompili ${ }^{a, b}$ (D), G. Pugliese $^{a, c}$ (D), D. Ramos ${ }^{a}$, A. Ranieri ${ }^{a}$ (D), G. Selvaggi ${ }^{a, b}$ (D) L. Silvestris ${ }^{a}$ (D), F.M. Simone ${ }^{a, b}$ (D), Ü. Sözbilir ${ }^{a}$, R. Venditti ${ }^{a}$ (D), P. Verwilligen ${ }^{a}$ (D)

INFN Sezione di Bologna ${ }^{a}$, Bologna, Italy, Università di Bologna ${ }^{b}$, Bologna, Italy

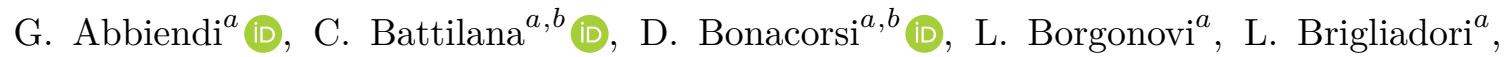
R. Campanini ${ }^{a, b}$ (D) P. Capiluppi ${ }^{a, b}$ (D) A. Castro ${ }^{a, b}$ (D), F.R. Cavallo ${ }^{a}$ (D) M. Cuffiani ${ }^{a, b}$ (D), G.M. Dallavalle ${ }^{a}$ (D), T. Diotalevi ${ }^{a, b}$ (D) F. Fabbri ${ }^{a}$ (D), A. Fanfani ${ }^{a, b}$ (D) P. Giacomelli ${ }^{a}$ (D), L. $\operatorname{Giommi}^{a, b}$ (D), C. Grandi ${ }^{a}$ (D), L. Guiducci ${ }^{a, b}$, S. Lo Meo ${ }^{a, 42}$, L. Lunerti ${ }^{a, b}$, S. Marcellini ${ }^{a}$ (D) G. Masetti ${ }^{a}$ (D) F.L. Navarria ${ }^{a, b}$ (D) A. Perrotta ${ }^{a}$ (D), F. Primavera ${ }^{a, b}$ (D), A.M. Rossi ${ }^{a, b}$ (D) T. Rovelli ${ }^{a, b}$ (D) G.P. Siroli ${ }^{a, b}$ (D)

INFN Sezione di Catania ${ }^{a}$, Catania, Italy, Università di Catania ${ }^{b}$, Catania, Italy

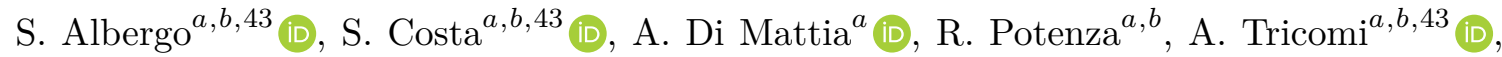

C. Tuve ${ }^{a, b}$ (D)

INFN Sezione di Firenze ${ }^{a}$, Firenze, Italy, Università di Firenze ${ }^{b}$, Firenze, Italy

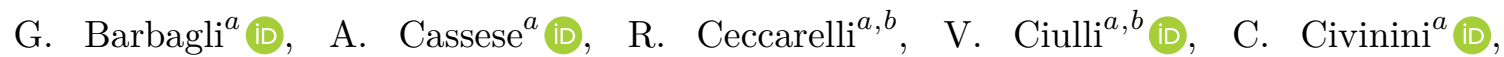
R. D'Alessandro ${ }^{a, b}$ (D) E. Focardi ${ }^{a, b}$ (D) G. Latino ${ }^{a, b}$ (D) P. Lenzi ${ }^{a, b}$ (D), M. Lizzo ${ }^{a, b}$, M. Meschini ${ }^{a}$ (D), S. Paoletti ${ }^{a}$ (D), R. Seidita ${ }^{a, b}$, G. Sguazzoni $^{a}$ (D) L. Viliani $^{a}$ (D)

INFN Laboratori Nazionali di Frascati, Frascati, Italy

L. Benussi (D), S. Bianco (D), D. Piccolo (D)

INFN Sezione di Genova ${ }^{a}$, Genova, Italy, Università di Genova ${ }^{b}$, Genova, Italy

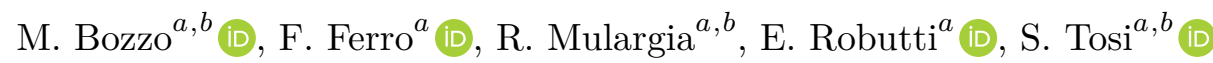


INFN Sezione di Milano-Bicocca ${ }^{a}$, Milano, Italy, Università di Milano-Bicocca , Milano, Italy

A. Benaglia ${ }^{a}$ (D), G. Boldrini (D), F. Brivio ${ }^{a, b}$, F. Cetorelli ${ }^{a, b}$, F. De Guio ${ }^{a, b}$ (D), M.E. $\operatorname{Dinardo}^{a, b}$ (D), P. $\operatorname{Dini}^{a}$ (D), S. Gennai ${ }^{a}\left(\mathbb{D}\right.$, A. Ghezzi $^{a, b}$ (D), P. Govoni ${ }^{a, b}$ (D),

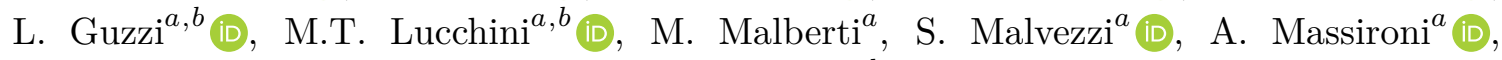

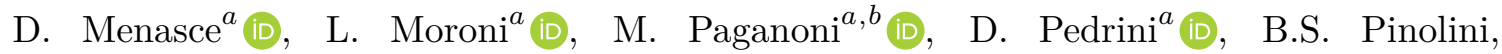
S. Ragazzi ${ }^{a, b}$ (D) N. Redaelli ${ }^{a}$ (D), T. Tabarelli de Fatis ${ }^{a, b}$ (D), D. Valsecchi ${ }^{a, b, 19}$, D. Zuolo $^{a, b}$ (D)

INFN Sezione di Napoli ${ }^{a}$, Napoli, Italy, Università di Napoli 'Federico II' ${ }^{b}$, Napoli, Italy, Università della Basilicata ${ }^{c}$, Potenza, Italy, Università G. Marconi ${ }^{d}$, Roma, Italy

S. Buontempo ${ }^{a}$ (D), F. Carnevali ${ }^{a, b}$, N. Cavallo ${ }^{a, c}$ (D), A. De Iorio ${ }^{a, b}$ (D) F. Fabozzi ${ }^{a, c}$ (D),

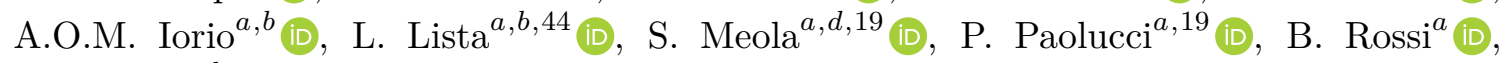
C. Sciacca ${ }^{a, b}$ iD

INFN Sezione di Padova ${ }^{a}$, Padova, Italy, Università di Padova ${ }^{b}$, Padova, Italy, Università di Trento ${ }^{c}$, Trento, Italy

P. $\operatorname{Azzi}^{a}$ (D), N. Bacchetta ${ }^{a}$ (D), D. Bisello ${ }^{a, b}$ (D), P. Bortignon ${ }^{a}$ (D), A. Bragagnolo ${ }^{a, b}$ (D),

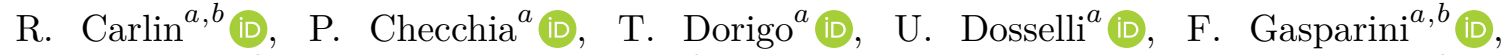
U. Gasparini ${ }^{a, b}$ (D) G. Grosso, S.Y. Hoh ${ }^{a, b}$ (D) L. Layer ${ }^{a, 45}$, E. Lusiani (D), M. Margoni ${ }^{a, b}$ (D), A.T. Meneguzzo ${ }^{a, b}$ (D) J. Pazzini ${ }^{a, b}$ (D), P. Ronchese ${ }^{a, b}$ (D), R. Rossin ${ }^{a, b}$, F. Simonetto ${ }^{a, b}$ (D),

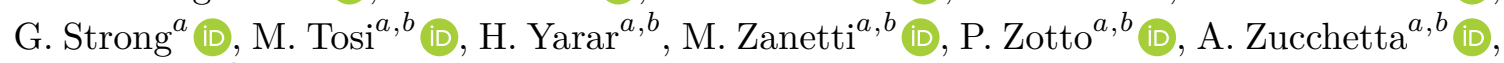
G. Zumerle ${ }^{a, b}$

INFN Sezione di Pavia ${ }^{a}$, Pavia, Italy, Università di Pavia ${ }^{b}$, Pavia, Italy C. Aime $^{a, b}$, A. Braghieri ${ }^{a}$ (D), S. Calzaferri ${ }^{a, b}$, D. Fiorina ${ }^{a, b}$ (D) P. Montagna ${ }^{a, b}$,

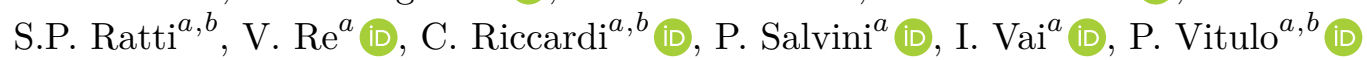

INFN Sezione di Perugia ${ }^{a}$, Perugia, Italy, Università di Perugia ${ }^{b}$, Perugia, Italy

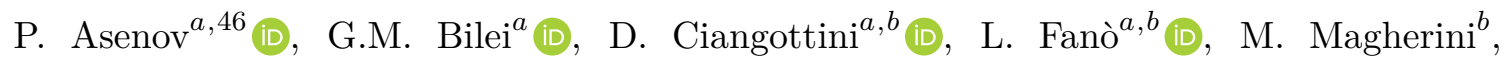
G. Mantovani $^{a, b}$, V. Mariani ${ }^{a, b}$, M. Menichelli ${ }^{a}$ (D), F. Moscatelli ${ }^{a, 46}$ (D) A. Piccinelli ${ }^{a, b}$ (D),

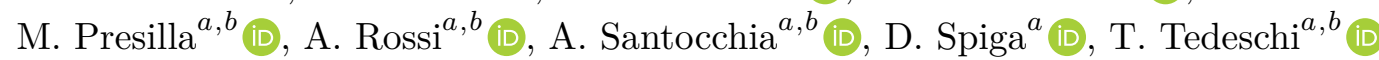

INFN Sezione di Pisa ${ }^{a}$, Pisa, Italy, Università di Pisa ${ }^{b}$, Pisa, Italy, Scuola Normale Superiore di Pisa ${ }^{c}$, Pisa, Italy, Università di Siena ${ }^{d}$, Siena, Italy

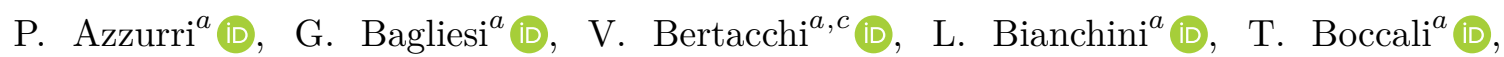
E. Bossini ${ }^{a, b}$ (D), R. Castaldi ${ }^{a}$ (D), M.A. Ciocci ${ }^{a, b}$ (D) V. D'Amante ${ }^{a, d}$ (D), R. Dell'Orso ${ }^{a}$ (D),

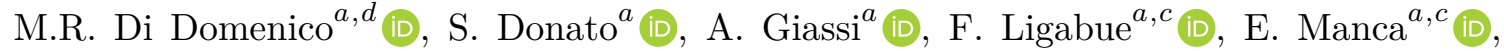
G. Mandorli ${ }^{a, c}$ (D), D. Matos Figueiredo, A. Messineo ${ }^{a, b}$ (D), F. Palla ${ }^{a}$ (D), S. Parolia ${ }^{a, b}$, G. Ramirez-Sanchez ${ }^{a, c}$, A. $\operatorname{Rizzi}^{a, b}$ (D), G. Rolandi ${ }^{a, c}$ (D), S. Roy Chowdhury ${ }^{a, c}$,

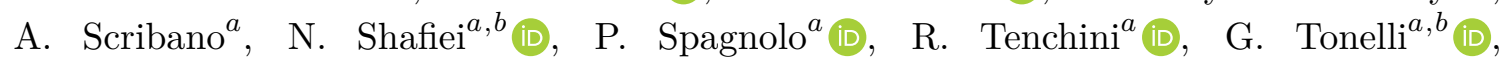

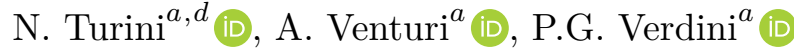


INFN Sezione di Roma ${ }^{a}$, Rome, Italy, Sapienza Università di Roma ${ }^{b}$, Rome, Italy

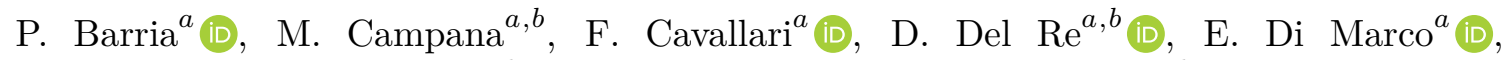

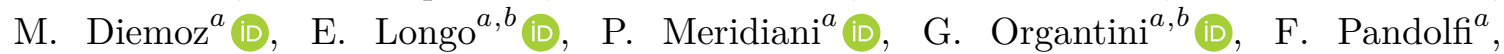

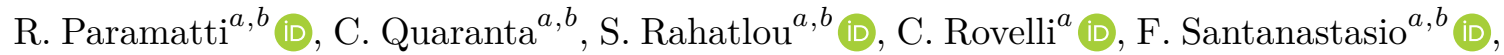
L. Soffi ${ }^{a}$ (D), R. Tramontano ${ }^{a, b}$

INFN Sezione di Torino ${ }^{a}$, Torino, Italy, Università di Torino ${ }^{b}$, Torino, Italy, Università del Piemonte Orientale ${ }^{c}$, Novara, Italy

N. Amapane ${ }^{a, b}$ (D) R. Arcidiacono ${ }^{a, c}$ (D), S. Argiro ${ }^{a, b}$ (D) M. Arneodo ${ }^{a, c}$ (D), N. Bartosik ${ }^{a}$ (D), R. Bellan ${ }^{a, b}$ (D), A. Bellora ${ }^{a, b}$ (D), J. Berenguer Antequera ${ }^{a, b}$ (D) C. Biino ${ }^{a}$ (D), N. Cartiglia ${ }^{a}$ (D),

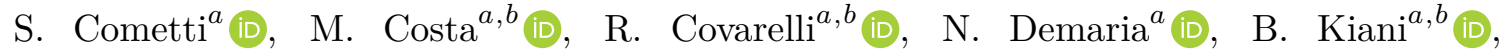

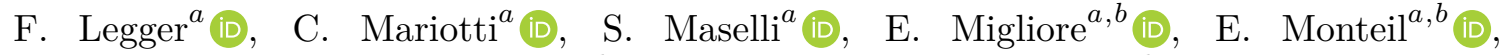

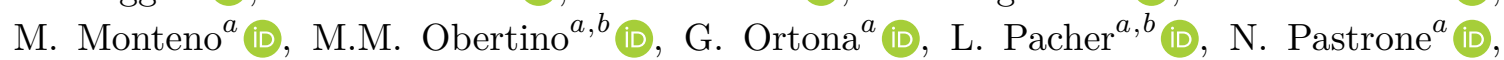
M. Pelliccioni ${ }^{a}$ (D), G.L. Pinna Angioni ${ }^{a, b}$, M. Ruspa $^{a, c}$ (D), K. Shchelina ${ }^{a}$ (D) F. Siviero ${ }^{a, b}$ (D),

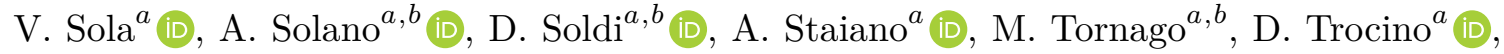
A. Vagnerini ${ }^{a, b}$

INFN Sezione di Trieste ${ }^{a}$, Trieste, Italy, Università di Trieste ${ }^{b}$, Trieste, Italy S. Belforte ${ }^{a}$ (D), V. Candelise ${ }^{a, b}$ (D), M. Casarsa ${ }^{a}$ (D), F. Cossutti ${ }^{a}$ (D), A. Da Rold ${ }^{a, b}$ (D), G. Della Ricca ${ }^{a, b}$ (D) G. Sorrentino ${ }^{a, b}$, F. Vazzoler ${ }^{a, b}$ (D)

Kyungpook National University, Daegu, Korea

S. Dogra (iD), C. Huh (iD, B. Kim, D.H. Kim (D), G.N. Kim (D), J. Kim, J. Lee, S.W. Lee(D), C.S. Moon (D), Y.D. Oh (D), S.I. Pak, B.C. Radburn-Smith, S. Sekmen (D), Y.C. Yang

Chonnam National University, Institute for Universe and Elementary Particles, Kwangju, Korea

H. Kim (D), D.H. Moon (D)

Hanyang University, Seoul, Korea

B. Francois (D), T.J. Kim (D), J. Park (D)

Korea University, Seoul, Korea

S. Cho, S. Choi (D), Y. Go, B. Hong (D), K. Lee, K.S. Lee (D), J. Lim, J. Park, S.K. Park, J. Yoo

Kyung Hee University, Department of Physics, Seoul, Republic of Korea, Seoul, Korea

J. Goh (D), A. Gurtu

Sejong University, Seoul, Korea

H.S. Kim (D), Y. Kim

\section{Seoul National University, Seoul, Korea}

J. Almond, J.H. Bhyun, J. Choi, S. Jeon, J. Kim, J.S. Kim, S. Ko, H. Kwon, H. Lee (D), S. Lee, B.H. Oh, M. Oh (iD, S.B. Oh, H. Seo (D), U.K. Yang, I. Yoon (iD 
University of Seoul, Seoul, Korea

W. Jang, D.Y. Kang, Y. Kang, S. Kim, B. Ko, J.S.H. Lee (D), Y. Lee, J.A. Merlin, I.C. Park, Y. Roh, M.S. Ryu, D. Song, I.J. Watson (D), S. Yang

Yonsei University, Department of Physics, Seoul, Korea

S. Ha, H.D. Yoo

Sungkyunkwan University, Suwon, Korea

M. Choi, H. Lee, Y. Lee, I. Yu

College of Engineering and Technology, American University of the Middle East (AUM), Egaila, Kuwait, Dasman, Kuwait

T. Beyrouthy, Y. Maghrbi

Riga Technical University, Riga, Latvia

K. Dreimanis (D), V. Veckalns ${ }^{47}$

Vilnius University, Vilnius, Lithuania

M. Ambrozas, A. Carvalho Antunes De Oliveira (D), A. Juodagalvis (ID, A. Rinkevicius (ID,

G. Tamulaitis (iD)

National Centre for Particle Physics, Universiti Malaya, Kuala Lumpur, Malaysia

N. Bin Norjoharuddeen (D, W.A.T. Wan Abdullah, M.N. Yusli, Z. Zolkapli

Universidad de Sonora (UNISON), Hermosillo, Mexico

J.F. Benitez (D), A. Castaneda Hernandez (D), M. León Coello, J.A. Murillo Quijada (D), A. Sehrawat, L. Valencia Palomo

Centro de Investigacion y de Estudios Avanzados del IPN, Mexico City, Mexico G. Ayala, H. Castilla-Valdez, E. De La Cruz-Burelo (D), I. Heredia-De La Cruz ${ }^{48}$ (D), R. Lopez-Fernandez, C.A. Mondragon Herrera, D.A. Perez Navarro, A. Sánchez Hernán$\operatorname{dez}(10)$

Universidad Iberoamericana, Mexico City, Mexico

S. Carrillo Moreno, C. Oropeza Barrera (D), F. Vazquez Valencia

Benemerita Universidad Autonoma de Puebla, Puebla, Mexico

I. Pedraza, H.A. Salazar Ibarguen, C. Uribe Estrada

University of Montenegro, Podgorica, Montenegro

J. Mijuskovic ${ }^{49}$, N. Raicevic

University of Auckland, Auckland, New Zealand

D. Krofcheck

University of Canterbury, Christchurch, New Zealand

P.H. Butler 
National Centre for Physics, Quaid-I-Azam University, Islamabad, Pakistan

A. Ahmad, M.I. Asghar, A. Awais, M.I.M. Awan, H.R. Hoorani, W.A. Khan, M.A. Shah, M. Shoaib (D), M. Waqas (iD)

AGH University of Science and Technology Faculty of Computer Science, Electronics and Telecommunications, Krakow, Poland

V. Avati, L. Grzanka, M. Malawski

National Centre for Nuclear Research, Swierk, Poland

H. Bialkowska, M. Bluj (D), B. Boimska (D), M. Górski, M. Kazana, M. Szleper (D), P. Zalewski

Institute of Experimental Physics, Faculty of Physics, University of Warsaw, Warsaw, Poland

K. Bunkowski, K. Doroba, A. Kalinowski (D), M. Konecki (D), J. Krolikowski (D)

Laboratório de Instrumentação e Física Experimental de Partículas, Lisboa, Portugal

M. Araujo, P. Bargassa (D), D. Bastos, A. Boletti (D), P. Faccioli (D), M. Gallinaro (D), J. Hollar (D), N. Leonardo (D), T. Niknejad, M. Pisano, J. Seixas (D), O. Toldaiev (D), J. Varela (D)

Joint Institute for Nuclear Research, Dubna, Russia

S. Afanasiev, D. Budkouski, I. Golutvin, I. Gorbunov (D), V. Karjavine, V. Korenkov (D), A. Lanev, A. Malakhov, V. Matveev ${ }^{50,51}$, V. Palichik, V. Perelygin, M. Savina, D. Seitova, V. Shalaev, S. Shmatov, S. Shulha, V. Smirnov, O. Teryaev, N. Voytishin, B.S. Yuldashev ${ }^{52}$,

A. Zarubin, I. Zhizhin

Petersburg Nuclear Physics Institute, Gatchina (St. Petersburg), Russia

G. Gavrilov (D), V. Golovtcov, Y. Ivanov, V. Kim ${ }^{53}$ (D), E. Kuznetsova ${ }^{54}$, V. Murzin, V. Oreshkin, I. Smirnov, D. Sosnov (D), V. Sulimov, L. Uvarov, S. Volkov, A. Vorobyev

Institute for Nuclear Research, Moscow, Russia

Yu. Andreev (D), A. Dermenev, S. Gninenko (D), N. Golubev, A. Karneyeu (D, D. Kirpichnikov (D), M. Kirsanov, N. Krasnikov, A. Pashenkov, G. Pivovarov (D), A. Toropin

Institute for Theoretical and Experimental Physics named by A.I. Alikhanov of NRC 'Kurchatov Institute', Moscow, Russia

V. Epshteyn, V. Gavrilov, N. Lychkovskaya, A. Nikitenko ${ }^{55}$, V. Popov, A. Stepennov, M. Toms, E. Vlasov (D), A. Zhokin

Moscow Institute of Physics and Technology, Moscow, Russia

T. Aushev

National Research Nuclear University 'Moscow Engineering Physics Institute' (MEPhI), Moscow, Russia

O. Bychkova, M. Chadeeva ${ }^{56}$ (D), A. Oskin, P. Parygin, S. Polikarpov ${ }^{57}$ (D), E. Popova

P.N. Lebedev Physical Institute, Moscow, Russia

V. Andreev, M. Azarkin, I. Dremin (D), M. Kirakosyan, A. Terkulov 
Skobeltsyn Institute of Nuclear Physics, Lomonosov Moscow State University, Moscow, Russia

A. Belyaev, E. Boos (D), V. Bunichev, M. Dubinin ${ }^{58}$ (D), L. Dudko (D), V. Klyukhin (D),

O. Kodolova (D), N. Korneeva (D), I. Lokhtin (D), S. Obraztsov, M. Perfilov, V. Savrin, P. Volkov

Novosibirsk State University (NSU), Novosibirsk, Russia

V. Blinov ${ }^{59}$, T. Dimova ${ }^{59}$, L. Kardapoltsev ${ }^{59}$, A. Kozyrev ${ }^{59}$, I. Ovtin ${ }^{59}$, O. Radchenko ${ }^{59}$, Y. Skovpen ${ }^{59}$ (D)

Institute for High Energy Physics of National Research Centre 'Kurchatov Institute', Protvino, Russia

I. Azhgirey (D), I. Bayshev, D. Elumakhov, V. Kachanov, D. Konstantinov (D), P. Mandrik (D), V. Petrov, R. Ryutin, S. Slabospitskii (D), A. Sobol, S. Troshin (D), N. Tyurin, A. Uzunian, A. Volkov

National Research Tomsk Polytechnic University, Tomsk, Russia

A. Babaev, V. Okhotnikov

Tomsk State University, Tomsk, Russia

V. Borshch, V. Ivanchenko (D), E. Tcherniaev (i)

University of Belgrade: Faculty of Physics and VINCA Institute of Nuclear Sciences, Belgrade, Serbia

P. Adzic ${ }^{60}$ (D), M. Dordevic (D), P. Milenovic (D), J. Milosevic (D)

Centro de Investigaciones Energéticas Medioambientales y Tecnológicas (CIEMAT), Madrid, Spain

M. Aguilar-Benitez, J. Alcaraz Maestre (D), A. Álvarez Fernández, I. Bachiller, M. Barrio Luna, Cristina F. Bedoya (D), C.A. Carrillo Montoya (D), M. Cepeda (D), M. Cerrada, N. Colino (D), B. De La Cruz, A. Delgado Peris (D), J.P. Fernández Ramos (D), J. Flix (D), M.C. Fouz (D), O. Gonzalez Lopez (D), S. Goy Lopez (D), J.M. Hernandez (D), M.I. Josa (D), J. León Holgado (D), D. Moran, Á. Navarro Tobar (D), C. Perez Dengra, A. PérezCalero Yzquierdo (D), J. Puerta Pelayo (D), I. Redondo (D), L. Romero, S. Sánchez Navas, L. Urda Gómez (D), C. Willmott

Universidad Autónoma de Madrid, Madrid, Spain

J.F. de Trocóniz, R. Reyes-Almanza (P)

Universidad de Oviedo, Instituto Universitario de Ciencias y Tecnologías Espaciales de Asturias (ICTEA), Oviedo, Spain

B. Alvarez Gonzalez (D), J. Cuevas (D), C. Erice (D), J. Fernandez Menendez (D), S. Folgueras (D), I. Gonzalez Caballero (D), J.R. González Fernández, E. Palencia Cortezon (D), C. Ramón Álvarez, V. Rodríguez Bouza (iD, A. Soto Rodríguez, A. Trapote, N. Trevisani (iD, C. Vico Villalba 
Instituto de Física de Cantabria (IFCA), CSIC-Universidad de Cantabria, Santander, Spain

J.A. Brochero Cifuentes (D), I.J. Cabrillo, A. Calderon (D), J. Duarte Campderros (D), M. Fernandez (D), C. Fernandez Madrazo (D), P.J. Fernández Manteca (D), A. García Alonso, G. Gomez, C. Martinez Rivero, P. Martinez Ruiz del Arbol (D), F. Matorras (D), P. Matorras Cuevas (D), J. Piedra Gomez (ID, C. Prieels, T. Rodrigo (D), A. Ruiz-Jimeno (D), L. Scodellaro (D), I. Vila, J.M. Vizan Garcia (DD

University of Colombo, Colombo, Sri Lanka

M.K. Jayananda, B. Kailasapathy ${ }^{61}$, D.U.J. Sonnadara, D.D.C. Wickramarathna

University of Ruhuna, Department of Physics, Matara, Sri Lanka

W.G.D. Dharmaratna (D), K. Liyanage, N. Perera, N. Wickramage

CERN, European Organization for Nuclear Research, Geneva, Switzerland

T.K. Aarrestad (D), D. Abbaneo, J. Alimena (D), E. Auffray, G. Auzinger, J. Baechler, P. Baillon ${ }^{\dagger}$, D. Barney (D), J. Bendavid, M. Bianco (D), A. Bocci (D), T. Camporesi, M. Capeans Garrido (D), G. Cerminara, N. Chernyavskaya (D), S.S. Chhibra (D), M. Cipriani (D), L. Cristella (D), D. d'Enterria (D), A. Dabrowski (D), A. David (D), A. De Roeck (D), M.M. Defranchis (D), M. Deile (D), M. Dobson, M. Dünser (D), N. Dupont, A. ElliottPeisert, N. Emriskova, F. Fallavollita ${ }^{62}$, A. Florent (D), G. Franzoni (D), W. Funk, S. Giani, D. Gigi, K. Gill, F. Glege, L. Gouskos (D), M. Haranko (D), J. Hegeman (D), V. Innocente (D), T. James, P. Janot (D), J. Kaspar (D), J. Kieseler (D), M. Komm (D), N. Kratochwil, C. Lange (D), S. Laurila, P. Lecoq (D), A. Lintuluoto, K. Long (D), C. Lourenço (D), B. Maier, L. Malgeri (D), S. Mallios, M. Mannelli, A.C. Marini (D), F. Meijers, S. Mersi (D), E. Meschi (D), F. Moortgat (D), M. Mulders (D), S. Orfanelli, L. Orsini, F. Pantaleo (D), E. Perez, M. Peruzzi (D), A. Petrilli, G. Petrucciani (D), A. Pfeiffer (D), M. Pierini (D), D. Piparo, M. Pitt (D), H. Qu (D), T. Quast, D. Rabady (D), A. Racz, G. Reales Gutiérrez, M. Rovere, H. Sakulin, J. SalfeldNebgen (D), S. Scarfi, C. Schäfer, C. Schwick, M. Selvaggi (D), A. Sharma, P. Silva (D), W. Snoeys (D), P. Sphicas ${ }^{63}$ (D), S. Summers (D), K. Tatar (D), V.R. Tavolaro (D), D. Treille, P. Tropea, A. Tsirou, G.P. Van Onsem (D), J. Wanczyk ${ }^{64}$, K.A. Wozniak, W.D. Zeuner

\section{Paul Scherrer Institut, Villigen, Switzerland}

L. Caminada ${ }^{65}$ (D) A. Ebrahimi (D), W. Erdmann, R. Horisberger, Q. Ingram, H.C. Kaestli, D. Kotlinski, U. Langenegger, M. Missiroli ${ }^{65}$ (D) L. Noehte ${ }^{65}$, T. Rohe

ETH Zurich - Institute for Particle Physics and Astrophysics (IPA), Zurich, Switzerland

K. Androsov ${ }^{64}$ (D), M. Backhaus (D), P. Berger, A. Calandri (D), A. De Cosa, G. Dissertori (D), M. Dittmar, M. Donegà, C. Dorfer (D), F. Eble, K. Gedia, F. Glessgen, T.A. Gómez Espinosa (iD, C. Grab (D), D. Hits, W. Lustermann, A.-M. Lyon, R.A. Manzoni (D), L. Marchese (D), C. Martin Perez, M.T. Meinhard, F. Nessi-Tedaldi, J. Niedziela (D), F. Pauss, V. Perovic, S. Pigazzini (D), M.G. Ratti (D), M. Reichmann, C. Reissel, T. Reitenspiess, B. Ristic (D), D. Ruini, D.A. Sanz Becerra (D), V. Stampf, J. Steggemann ${ }^{64}$ (D), R. Wallny (D), D.H. Zhu 
Universität Zürich, Zurich, Switzerland

C. Amsler ${ }^{66}$ (D) P. Bärtschi, C. Botta (D), D. Brzhechko, M.F. Canelli (D), K. Cormier, A. De Wit (D), R. Del Burgo, J.K. Heikkilä (D), M. Huwiler, W. Jin, A. Jofrehei (D), B. Kilminster (D), S. Leontsinis (D), S.P. Liechti, A. Macchiolo (D), P. Meiring, V.M. Mikuni (D), U. Molinatti, I. Neutelings, A. Reimers, P. Robmann, S. Sanchez Cruz (D), K. Schweiger (D), M. Senger, Y. Takahashi (D)

National Central University, Chung-Li, Taiwan

C. Adloff ${ }^{67}$, C.M. Kuo, W. Lin, A. Roy (D), T. $\operatorname{Sarkar}^{36}$ (D), S.S. Yu

National Taiwan University (NTU), Taipei, Taiwan

L. Ceard, Y. Chao, K.F. Chen (D), P.H. Chen (D), W.-S. Hou (D), Y.y. Li, R.-S. Lu, E. Paganis (D), A. Psallidas, A. Steen, H.y. Wu, E. Yazgan (D), P.r. Yu

Chulalongkorn University, Faculty of Science, Department of Physics, Bangkok, Thailand

B. Asavapibhop (D), C. Asawatangtrakuldee (D), N. Srimanobhas (D)

Çukurova University, Physics Department, Science and Art Faculty, Adana, Turkey

F. Boran (D), S. Damarseckin ${ }^{68}$, Z.S. Demiroglu (D, F. Dolek (D) I. Dumanoglu ${ }^{69}$ (D), E. Eskut, Y. Guler ${ }^{70}$ (D), E. Gurpinar Guler ${ }^{70}$ (D), C. Isik, O. Kara, A. Kayis Topaksu, U. Kiminsu (D), G. Onengut, K. Ozdemir ${ }^{71}$, A. Polatoz, A.E. Simsek (D), B. Tali ${ }^{72}$, U.G. Tok (D), S. Turkcapar, I.S. Zorbakir if

Middle East Technical University, Physics Department, Ankara, Turkey

B. Isildak ${ }^{73}$, G. Karapinar, K. Ocalan ${ }^{74}$ (D), M. Yalvac ${ }^{75}$

Bogazici University, Istanbul, Turkey

B. Akgun, I.O. Atakisi (D), E. Gülmez (D), M. Kaya ${ }^{76}$ (D) O. Kaya ${ }^{77}$, Ö. Özçelik, S. Tekten $^{78}$, E.A. Yetkin ${ }^{79}$ (D)

Istanbul Technical University, Istanbul, Turkey

A. Cakir (D), K. Cankocak ${ }^{69}$ (D), Y. Komurcu, S. $\operatorname{Sen}^{80}$ (D)

Istanbul University, Istanbul, Turkey

S. Cerci ${ }^{72}$, I. $\operatorname{Hos}^{81}$, B. Kaynak, S. Ozkorucuklu, H. Sert (D), D. Sunar Cerci ${ }^{72}$ (D), C. Zorbilmez

Institute for Scintillation Materials of National Academy of Science of Ukraine, Kharkov, Ukraine

B. Grynyov

National Scientific Center, Kharkov Institute of Physics and Technology, Kharkov, Ukraine

L. Levchuk 


\section{University of Bristol, Bristol, United Kingdom}

D. Anthony, E. Bhal (D), S. Bologna, J.J. Brooke (D), A. Bundock (D), E. Clement (D),

D. Cussans (D), H. Flacher (D), J. Goldstein (D), G.P. Heath, H.F. Heath (D), L. Kreczko (D),

B. Krikler (D), S. Paramesvaran, S. Seif El Nasr-Storey, V.J. Smith, N. Stylianou ${ }^{82}$ (D),

K. Walkingshaw Pass, R. White

\section{Rutherford Appleton Laboratory, Didcot, United Kingdom}

K.W. Bell, A. Belyaev ${ }^{83}$ (D), C. Brew (D), R.M. Brown, D.J.A. Cockerill, C. Cooke, K.V. Ellis, K. Harder, S. Harper, M.-L. Holmberg ${ }^{84}$, J. Linacre (D), K. Manolopoulos, D.M. Newbold (ID, E. Olaiya, D. Petyt, T. Reis (D), T. Schuh, C.H. Shepherd-Themistocleous, I.R. Tomalin, T. Williams (D)

\section{Imperial College, London, United Kingdom}

R. Bainbridge (ID, P. Bloch (D), S. Bonomally, J. Borg (D), S. Breeze, O. Buchmuller, V. Cepaitis (D), G.S. Chahal ${ }^{85}$ (D), D. Colling, P. Dauncey (D), G. Davies (D), M. Della Negra (D), S. Fayer, G. Fedi (D, G. Hall (D), M.H. Hassanshahi, G. Iles, J. Langford, L. Lyons, A.M. Magnan, S. Malik, A. Martelli (D), D.G. Monk, J. Nash ${ }^{86}$ (D), M. Pesaresi, D.M. Raymond, A. Richards, A. Rose, E. Scott (D), C. Seez, A. Shtipliyski, A. Tapper (D), K. Uchida, T. Virdee ${ }^{19}$ (D), M. Vojinovic (D), N. Wardle (D), S.N. Webb (D), D. Winterbottom

\section{Brunel University, Uxbridge, United Kingdom}

K. Coldham, J.E. Cole (D), A. Khan, P. Kyberd (D), I.D. Reid (D), L. Teodorescu, S. Zahid (D)

Baylor University, Waco, Texas, U.S.A.

S. Abdullin (D), A. Brinkerhoff(D), B. Caraway (D), J. Dittmann (D), K. Hatakeyama (D), A.R. Kanuganti, B. McMaster (D), N. Pastika, M. Saunders (D), S. Sawant, C. Sutantawibul, J. Wilson (iD)

Catholic University of America, Washington, DC, U.S.A.

R. Bartek (D), A. Dominguez (D), R. Uniyal (D), A.M. Vargas Hernandez

The University of Alabama, Tuscaloosa, Alabama, U.S.A.

A. Buccilli (D), S.I. Cooper (D), D. Di Croce (D), S.V. Gleyzer (D), C. Henderson (D), C.U. Perez (D), P. Rumerio ${ }^{87}$ (D), C. West (D)

Boston University, Boston, Massachusetts, U.S.A.

A. Akpinar (D), A. Albert (D), D. Arcaro (D), C. Cosby (D), Z. Demiragli (D), E. Fontanesi, D. Gastler, S. May (D), J. Rohlf (ID, K. Salyer (D), D. Sperka, D. Spitzbart (D), I. Suarez (D), A. Tsatsos, S. Yuan, D. Zou

Brown University, Providence, Rhode Island, U.S.A.

G. Benelli (D), B. Burkle (D), X. Coubez ${ }^{20}$, D. Cutts (D), M. Hadley (D), U. Heintz (D), J.M. $\operatorname{Hogan}^{88}$ (D), T. KWON, G. Landsberg (D), K.T. Lau (D), D. Li, M. Lukasik, J. Luo (D), M. Narain, N. Pervan, S. Sagir ${ }^{89}$ (D), F. Simpson, E. Usai (D), W.Y. Wong, X. Yan (D), D. Yu (iD, W. Zhang 
University of California, Davis, Davis, California, U.S.A.

J. Bonilla (D), C. Brainerd (D), R. Breedon, M. Calderon De La Barca Sanchez, M. Chertok (ID,

J. Conway (D), P.T. Cox, R. Erbacher, G. Haza, F. Jensen (D), O. Kukral, R. Lander, M. Mulhearn (D), D. Pellett, B. Regnery (D), D. Taylor (D), Y. Yao (D), F. Zhang (D)

University of California, Los Angeles, California, U.S.A.

M. Bachtis (D), R. Cousins (D), A. Datta (D), D. Hamilton, J. Hauser (D), M. Ignatenko, M.A. Iqbal, T. Lam, W.A. Nash, S. Regnard (D), D. Saltzberg (D), B. Stone, V. Valuev (D)

University of California, Riverside, Riverside, California, U.S.A.

K. Burt, Y. Chen, R. Clare (D), J.W. Gary (D), M. Gordon, G. Hanson (D), G. Karapostoli (D), O.R. Long (D), N. Manganelli, M. Olmedo Negrete, W. Si (D), S. Wimpenny, Y. Zhang

University of California, San Diego, La Jolla, California, U.S.A.

J.G. Branson, P. Chang (D), S. Cittolin, S. Cooperstein (D), N. Deelen (D), D. Diaz (D), J. Duarte (D), R. Gerosa (D), L. Giannini (D), J. Guiang, R. Kansal (D), V. Krutelyov (D), R. Lee, J. Letts (D), M. Masciovecchio (D), F. Mokhtar, M. Pieri (D), B.V. Sathia Narayanan (D), V. Sharma (D), M. Tadel, A. Vartak (D), F. Würthwein (D), Y. Xiang (D), A. Yagil (D)

University of California, Santa Barbara - Department of Physics, Santa Barbara, California, U.S.A.

N. Amin, C. Campagnari (D, M. Citron (D), A. Dorsett, V. Dutta (D), J. Incandela (D), M. Kilpatrick (D), J. Kim (D), B. Marsh, H. Mei, M. Oshiro, M. Quinnan (D), J. Richman, U. Sarica (D), F. Setti, J. Sheplock, P. Siddireddy, D. Stuart, S. Wang (D)

California Institute of Technology, Pasadena, California, U.S.A.

A. Bornheim (D), O. Cerri, I. Dutta (D), J.M. Lawhorn (D), N. Lu (D), J. Mao, H.B. Newman (D), T.Q. Nguyen (D), M. Spiropulu (D), J.R. Vlimant (D), C. Wang (D), S. Xie (D), Z. Zhang (D), R.Y. Zhu (D)

Carnegie Mellon University, Pittsburgh, Pennsylvania, U.S.A.

J. Alison (D), S. An (D), M.B. Andrews, P. Bryant (D), T. Ferguson (D), A. Harilal, C. Liu, T. Mudholkar (D), M. Paulini (D), A. Sanchez, W. Terrill

University of Colorado Boulder, Boulder, Colorado, U.S.A.

J.P. Cumalat (D, W.T. Ford (D), A. Hassani, E. MacDonald, R. Patel, A. Perloff(D), C. Savard, K. Stenson (D), K.A. Ulmer (D), S.R. Wagner (D)

Cornell University, Ithaca, New York, U.S.A.

J. Alexander (D), S. Bright-Thonney (D), X. Chen (D), Y. Cheng (D), D.J. Cranshaw (D), S. Hogan, J. Monroy (D), J.R. Patterson (D), D. Quach (D), J. Reichert (D), M. Reid (D), A. Ryd, W. Sun (D), J. Thom (D), P. Wittich (D), R. Zou (D)

Fermi National Accelerator Laboratory, Batavia, Illinois, U.S.A.

M. Albrow (D), M. Alyari (D), G. Apollinari, A. Apresyan (D), A. Apyan (D), S. Banerjee, L.A.T. Bauerdick (D), D. Berry (D), J. Berryhill (D), P.C. Bhat, K. Burkett (D), J.N. Butler, A. Canepa, G.B. Cerati (D), H.W.K. Cheung (D), F. Chlebana, K.F. Di Petrillo (D), V.D. Elvira (D), Y. Feng, J. Freeman, Z. Gecse, L. Gray, D. Green, S. Grünendahl (D), 
O. Gutsche (D), R.M. Harris (D), R. Heller, T.C. Herwig (D), J. Hirschauer (D), B. Jayatilaka (D), S. Jindariani, M. Johnson, U. Joshi, T. Klijnsma (D), B. Klima (D), K.H.M. Kwok, S. Lammel (D), D. Lincoln (D), R. Lipton, T. Liu, C. Madrid, K. Maeshima, C. Mantilla (D), D. Mason, P. McBride (D), P. Merkel, S. Mrenna (D), S. Nahn (D), J. Ngadiuba (D), V. O'Dell, V. Papadimitriou, K. Pedro (D), C. Pena ${ }^{58}$ (D), O. Prokofyev, F. Ravera (D), A. Reinsvold Hall (D), L. Ristori (D), E. Sexton-Kennedy (D), N. Smith (D), A. Soha (D), W.J. Spalding (D), L. Spiegel, S. Stoynev (D), J. Strait (D), L. Taylor (D), S. Tkaczyk, N.V. Tran (D), L. Uplegger (D), E.W. Vaandering (D), H.A. Weber (D)

University of Florida, Gainesville, Florida, U.S.A.

D. Acosta (D), P. Avery, D. Bourilkov (D), L. Cadamuro (D), V. Cherepanov, F. Errico (D), R.D. Field, D. Guerrero, B.M. Joshi (D), M. Kim, E. Koenig, J. Konigsberg (D), A. Korytov, K.H. Lo, K. Matchev (D), N. Menendez (D), G. Mitselmakher (D), A. Muthirakalayil Madhu, N. Rawal, D. Rosenzweig, S. Rosenzweig, J. Rotter, K. Shi (D), J. Sturdy (D), J. Wang (D), E. Yigitbasi (D), X. Zuo

Florida State University, Tallahassee, Florida, U.S.A.

T. Adams (D), A. Askew (D), R. Habibullah (D), V. Hagopian, K.F. Johnson, R. Khurana, T. Kolberg (iD, G. Martinez, H. Prosper (D), C. Schiber, O. Viazlo (iD, R. Yohay (D), J. Zhang

Florida Institute of Technology, Melbourne, Florida, U.S.A.

M.M. Baarmand (D), S. Butalla, T. Elkafrawy ${ }^{90}$ (D), M. Hohlmann (D), R. Kumar Verma (D), D. Noonan (D), M. Rahmani, F. Yumiceva (D)

University of Illinois at Chicago (UIC), Chicago, Illinois, U.S.A.

M.R. Adams, H. Becerril Gonzalez (D), R. Cavanaugh (D), S. Dittmer, O. Evdokimov (D), C.E. Gerber (D), D.A. Hangal (D), D.J. Hofman (D), A.H. Merrit, C. Mills (D), G. Oh (D), T. Roy, S. Rudrabhatla, M.B. Tonjes (D), N. Varelas (D), J. Viinikainen (D), X. Wang, Z. Wu (D), Z. Ye (iD

The University of Iowa, Iowa City, Iowa, U.S.A.

M. Alhusseini (D), K. Dilsiz ${ }^{91}$ (D) L. Emediato, R.P. Gandrajula (D), O.K. Köseyan (D), J.P. Merlo, A. Mestvirishvili ${ }^{92}$, J. Nachtman, H. Ogul ${ }^{93}$ (D) Y. Onel (D), A. Penzo, C. Snyder, E. $\operatorname{Tiras}^{94}(1)$

Johns Hopkins University, Baltimore, Maryland, U.S.A.

O. Amram (D), B. Blumenfeld (D), L. Corcodilos (D), J. Davis, M. Eminizer (D), A.V. Gritsan (D), S. Kyriacou, P. Maksimovic (D), J. Roskes (D), M. Swartz, T.Á. Vámi (D)

The University of Kansas, Lawrence, Kansas, U.S.A.

A. Abreu, J. Anguiano, C. Baldenegro Barrera (D), P. Baringer (D), A. Bean (D), A. Bylinkin (D), Z. Flowers, T. Isidori, S. Khalil (D), J. King, G. Krintiras (D), A. Kropivnitskaya (D), M. Lazarovits, C. Le Mahieu, C. Lindsey, J. Marquez, N. Minafra (D, M. Murray (D), M. Nickel, C. Rogan (D), C. Royon, R. Salvatico (D), S. Sanders, E. Schmitz, C. Smith (ID, J.D. Tapia Takaki (D), Q. Wang (D), Z. Warner, J. Williams (D), G. Wilson (ID 
Kansas State University, Manhattan, Kansas, U.S.A.

S. Duric, A. Ivanov (D), K. Kaadze (D), D. Kim, Y. Maravin (D), T. Mitchell, A. Modak, K. Nam

Lawrence Livermore National Laboratory, Livermore, California, U.S.A.

F. Rebassoo, D. Wright

University of Maryland, College Park, Maryland, U.S.A.

E. Adams, A. Baden, O. Baron, A. Belloni (iD, S.C. Eno (D), N.J. Hadley (iD, S. Jabeen (ID), R.G. Kellogg, T. Koeth, S. Lascio, A.C. Mignerey, S. Nabili, C. Palmer (D), M. Seidel (D), A. Skuja (D), L. Wang, K. Wong (D)

Massachusetts Institute of Technology, Cambridge, Massachusetts, U.S.A.

D. Abercrombie, G. Andreassi, R. Bi, W. Busza (D), I.A. Cali, Y. Chen (D), M. D'Alfonso (D),

J. Eysermans, C. Freer (D), G. Gomez Ceballos, M. Goncharov, P. Harris, M. Hu, M. Klute (D), D. Kovalskyi (D), J. Krupa, Y.-J. Lee(D), C. Mironov (D), C. Paus (D), D. Rankin (D), C. Roland (D), G. Roland, Z. Shi (D), G.S.F. Stephans (D), J. Wang, Z. Wang (D), B. Wyslouch (D)

University of Minnesota, Minneapolis, Minnesota, U.S.A.

R.M. Chatterjee, A. Evans (D), J. Hiltbrand, Sh. Jain (D), M. Krohn, Y. Kubota, J. Mans (D), M. Revering, R. Rusack (D), R. Saradhy, N. Schroeder (D), N. Strobbe (D), M.A. Wadud

University of Nebraska-Lincoln, Lincoln, Nebraska, U.S.A.

K. Bloom (D), M. Bryson, S. Chauhan (D), D.R. Claes, C. Fangmeier, L. Finco (D), F. Golf (D), C. Joo, I. Kravchenko (D), M. Musich, I. Reed, J.E. Siado, G.R. Snow ${ }^{\dagger}$, W. Tabb, F. Yan, A.G. Zecchinelli

State University of New York at Buffalo, Buffalo, New York, U.S.A.

G. Agarwal (D), H. Bandyopadhyay (D), L. Hay (D), I. Iashvili (D, A. Kharchilava, C. McLean (D), D. Nguyen, J. Pekkanen (D), S. Rappoccio (D), A. Williams (D)

Northeastern University, Boston, Massachusetts, U.S.A.

G. Alverson (D), E. Barberis, Y. Haddad (D), A. Hortiangtham, J. Li (D), G. Madigan, B. Marzocchi (D), D.M. Morse (D), V. Nguyen, T. Orimoto (D), A. Parker, L. Skinnari (D), A. Tishelman-Charny, T. Wamorkar, B. Wang (D), A. Wisecarver, D. Wood (D)

Northwestern University, Evanston, Illinois, U.S.A.

S. Bhattacharya (D), J. Bueghly, Z. Chen (D), A. Gilbert (D), T. Gunter (D), K.A. Hahn, Y. Liu, N. Odell, M.H. Schmitt (D), M. Velasco

University of Notre Dame, Notre Dame, Indiana, U.S.A.

R. Band (D), R. Bucci, M. Cremonesi, A. Das (D), N. Dev (D), R. Goldouzian (D), M. Hildreth, K. Hurtado Anampa (iD, C. Jessop (D), K. Lannon (D), J. Lawrence, N. Loukas (D), D. Lutton, J. Mariano, N. Marinelli, I. Mcalister, T. McCauley (D), C. Mcgrady, K. Mohrman, C. Moore, Y. Musienko ${ }^{50}$, R. Ruchti, A. Townsend, M. Wayne, A. Wightman, M. Zarucki (D), L. Zygala 
The Ohio State University, Columbus, Ohio, U.S.A.

B. Bylsma, B. Cardwell, L.S. Durkin (D), B. Francis (D), C. Hill (D), M. Nunez Ornelas (D), K. Wei, B.L. Winer, B.R. Yates (D)

Princeton University, Princeton, New Jersey, U.S.A.

F.M. Addesa (iD, B. Bonham (D), P. Das (D), G. Dezoort, P. Elmer (D), A. Frankenthal (D), B. Greenberg (D), N. Haubrich, S. Higginbotham, A. Kalogeropoulos (D), G. Kopp, S. Kwan (D), D. Lange, D. Marlow (D), K. Mei (D), I. Ojalvo, J. Olsen (D), D. Stickland (D), C. Tully (1D)

University of Puerto Rico, Mayaguez, Puerto Rico, U.S.A.

S. Malik (D), S. Norberg

Purdue University, West Lafayette, Indiana, U.S.A.

A.S. Bakshi, V.E. Barnes (D), R. Chawla (D), S. Das (D), L. Gutay, M. Jones (D), A.W. Jung (D), S. Karmarkar, D. Kondratyev (D), M. Liu, G. Negro, N. Neumeister (D), G. Paspalaki, S. Piperov (D), A. Purohit, J.F. Schulte (D), M. Stojanovic ${ }^{16}$, J. Thieman (D), F. Wang (D), R. Xiao (D), W. Xie (D)

Purdue University Northwest, Hammond, Indiana, U.S.A.

J. Dolen (D), N. Parashar

Rice University, Houston, Texas, U.S.A.

A. Baty (D), T. Carnahan, M. Decaro, S. Dildick (D), K.M. Ecklund (D), S. Freed, P. Gardner, F.J.M. Geurts (D), A. Kumar (D), W. Li, B.P. Padley (D), R. Redjimi, W. Shi (D), A.G. Stahl Leiton (D), S. Yang (D), L. Zhang ${ }^{95}$, Y. Zhang (D)

University of Rochester, Rochester, New York, U.S.A.

A. Bodek (D), P. de Barbaro, R. Demina (D), J.L. Dulemba (D), C. Fallon, T. Ferbel (D), M. Galanti, A. Garcia-Bellido (D), O. Hindrichs (ID, A. Khukhunaishvili, E. Ranken, R. Taus

Rutgers, The State University of New Jersey, Piscataway, New Jersey, U.S.A. B. Chiarito, J.P. Chou (D), A. Gandrakota (D), Y. Gershtein (D), E. Halkiadakis (D), A. Hart, M. Heindl (D), O. Karacheban ${ }^{23}$ (D), I. Laflotte, A. Lath (D), R. Montalvo, K. Nash, M. Osherson, S. Salur (D), S. Schnetzer, S. Somalwar (D), R. Stone, S.A. Thayil (D), S. Thomas, H. Wang (D)

University of Tennessee, Knoxville, Tennessee, U.S.A.

H. Acharya, A.G. Delannoy (D), S. Fiorendi (D), S. Spanier (D)

Texas A\&M University, College Station, Texas, U.S.A.

O. Bouhali ${ }^{96}$ (D) M. Dalchenko (D), A. Delgado (D), R. Eusebi, J. Gilmore, T. Huang, T. Kamon ${ }^{97}$, H. Kim (D), S. Luo (D), S. Malhotra, R. Mueller, D. Overton, D. Rathjens (D), A. Safonov (iD)

Texas Tech University, Lubbock, Texas, U.S.A.

N. Akchurin, J. Damgov, V. Hegde, S. Kunori, K. Lamichhane, S.W. Lee (D), T. Mengke, S. Muthumuni (iD, T. Peltola (ID, I. Volobouev, Z. Wang, A. Whitbeck 
Vanderbilt University, Nashville, Tennessee, U.S.A.

E. Appelt (D), S. Greene, A. Gurrola (D), W. Johns, A. Melo, H. Ni, K. Padeken (D), F. Romeo (D), P. Sheldon (D), S. Tuo, J. Velkovska (D)

\section{University of Virginia, Charlottesville, Virginia, U.S.A.}

M.W. Arenton (D), B. Cox (D), G. Cummings (D), J. Hakala (D), R. Hirosky (D), M. Joyce (D), A. Ledovskoy (iD, A. Li, C. Neu (D), C.E. Perez Lara (D), B. Tannenwald (D), S. White (D), E. Wolfe (iD

\section{Wayne State University, Detroit, Michigan, U.S.A.}

N. Poudyal (D)

University of Wisconsin - Madison, Madison, WI, Wisconsin, U.S.A.

K. Black (D), T. Bose (D), C. Caillol, S. Dasu (D), I. De Bruyn (D), P. Everaerts (D), F. Fienga (D), C. Galloni, H. He, M. Herndon (D), A. Hervé, U. Hussain, A. Lanaro, A. Loeliger, R. Loveless, J. Madhusudanan Sreekala (D), A. Mallampalli, A. Mohammadi, D. Pinna, A. Savin, V. Shang, V. Sharma (D), W.H. Smith (D), D. Teague, S. Trembath-Reichert, W. Vetens (i)

\section{t: Deceased}

1: Also at TU Wien, Wien, Austria

2: Also at Institute of Basic and Applied Sciences, Faculty of Engineering, Arab Academy for Science, Technology and Maritime Transport, Alexandria, Egypt

3: Also at Université Libre de Bruxelles, Bruxelles, Belgium

4: Also at Universidade Estadual de Campinas, Campinas, Brazil

5: Also at Federal University of Rio Grande do Sul, Porto Alegre, Brazil

6: Also at The University of the State of Amazonas, Manaus, Brazil

7: Also at University of Chinese Academy of Sciences, Beijing, China

8: Also at Department of Physics, Tsinghua University, Beijing, China

9: Also at UFMS, Nova Andradina, Brazil

10: Also at Nanjing Normal University Department of Physics, Nanjing, China

11: Now at The University of Iowa, Iowa City, Iowa, U.S.A.

12: Also at Institute for Theoretical and Experimental Physics named by A.I. Alikhanov of NRC 'Kurchatov Institute', Moscow, Russia

13: Also at Joint Institute for Nuclear Research, Dubna, Russia

14: Now at Cairo University, Cairo, Egypt

15: Also at Zewail City of Science and Technology, Zewail, Egypt

16: Also at Purdue University, West Lafayette, Indiana, U.S.A.

17: Also at Université de Haute Alsace, Mulhouse, France

18: Also at Erzincan Binali Yildirim University, Erzincan, Turkey

19: Also at CERN, European Organization for Nuclear Research, Geneva, Switzerland

20: Also at RWTH Aachen University, III. Physikalisches Institut A, Aachen, Germany

21: Also at University of Hamburg, Hamburg, Germany

22: Also at Isfahan University of Technology, Isfahan, Iran

23: Also at Brandenburg University of Technology, Cottbus, Germany

24: Also at Forschungszentrum Jülich, Juelich, Germany

25: Also at Physics Department, Faculty of Science, Assiut University, Assiut, Egypt 
26: Also at Karoly Robert Campus, MATE Institute of Technology, Gyongyos, Hungary

27: Also at Institute of Physics, University of Debrecen, Debrecen, Hungary

28: Also at Institute of Nuclear Research ATOMKI, Debrecen, Hungary

29: Also at MTA-ELTE Lendület CMS Particle and Nuclear Physics Group, Eötvös Loránd University, Budapest, Hungary

30: Also at Wigner Research Centre for Physics, Budapest, Hungary

31: Also at IIT Bhubaneswar, Bhubaneswar, India

32: Also at Institute of Physics, Bhubaneswar, India

33: Also at Punjab Agricultural University, Ludhiana, India

34: Also at Shoolini University, Solan, India

35: Also at University of Hyderabad, Hyderabad, India

36: Also at University of Visva-Bharati, Santiniketan, India

37: Also at Indian Institute of Technology (IIT), Mumbai, India

38: Also at Deutsches Elektronen-Synchrotron, Hamburg, Germany

39: Also at Sharif University of Technology, Tehran, Iran

40: Also at Department of Physics, University of Science and Technology of Mazandaran, Behshahr, Iran

41: Now at INFN Sezione di Bari, Università di Bari, Politecnico di Bari, Bari, Italy

42: Also at Italian National Agency for New Technologies, Energy and Sustainable Economic Development, Bologna, Italy

43: Also at Centro Siciliano di Fisica Nucleare e di Struttura Della Materia, Catania, Italy

44: Also at Scuola Superiore Meridionale, Università di Napoli Federico II, Napoli, Italy

45: Also at Università di Napoli 'Federico II', Napoli, Italy

46: Also at Consiglio Nazionale delle Ricerche - Istituto Officina dei Materiali, Perugia, Italy

47: Also at Riga Technical University, Riga, Latvia

48: Also at Consejo Nacional de Ciencia y Tecnología, Mexico City, Mexico

49: Also at IRFU, CEA, Université Paris-Saclay, Gif-sur-Yvette, France

50: Also at Institute for Nuclear Research, Moscow, Russia

51: Now at National Research Nuclear University 'Moscow Engineering Physics Institute' (MEPhI), Moscow, Russia

52: Also at Institute of Nuclear Physics of the Uzbekistan Academy of Sciences, Tashkent, Uzbekistan

53: Also at St. Petersburg Polytechnic University, St. Petersburg, Russia

54: Also at University of Florida, Gainesville, Florida, U.S.A.

55: Also at Imperial College, London, United Kingdom

56: Also at Moscow Institute of Physics and Technology, Moscow, Russia

57: Also at P.N. Lebedev Physical Institute, Moscow, Russia

58: Also at California Institute of Technology, Pasadena, California, U.S.A.

59: Also at Budker Institute of Nuclear Physics, Novosibirsk, Russia

60: Also at Faculty of Physics, University of Belgrade, Belgrade, Serbia

61: Also at Trincomalee Campus, Eastern University, Sri Lanka, Nilaveli, Sri Lanka

62: Also at INFN Sezione di Pavia, Università di Pavia, Pavia, Italy

63: Also at National and Kapodistrian University of Athens, Athens, Greece

64: Also at Ecole Polytechnique Fédérale Lausanne, Lausanne, Switzerland

65: Also at Universität Zürich, Zurich, Switzerland

66: Also at Stefan Meyer Institute for Subatomic Physics, Vienna, Austria

67: Also at Laboratoire d'Annecy-le-Vieux de Physique des Particules, IN2P3-CNRS, Annecyle-Vieux, France 
68: Also at Şırnak University, Sirnak, Turkey

69: Also at Near East University, Research Center of Experimental Health Science, Nicosia, Turkey

70: Also at Konya Technical University, Konya, Turkey

71: Also at Piri Reis University, Istanbul, Turkey

72: Also at Adiyaman University, Adiyaman, Turkey

73: Also at Ozyegin University, Istanbul, Turkey

74: Also at Necmettin Erbakan University, Konya, Turkey

75: Also at Bozok Universitetesi Rektörlügü, Yozgat, Turkey

76: Also at Marmara University, Istanbul, Turkey

77: Also at Milli Savunma University, Istanbul, Turkey

78: Also at Kafkas University, Kars, Turkey

79: Also at Istanbul Bilgi University, Istanbul, Turkey

80: Also at Hacettepe University, Ankara, Turkey

81: Also at Istanbul University - Cerrahpasa, Faculty of Engineering, Istanbul, Turkey

82: Also at Vrije Universiteit Brussel, Brussel, Belgium

83: Also at School of Physics and Astronomy, University of Southampton, Southampton, United Kingdom

84: Also at Rutherford Appleton Laboratory, Didcot, United Kingdom

85: Also at IPPP Durham University, Durham, United Kingdom

86: Also at Monash University, Faculty of Science, Clayton, Australia

87: Also at Università di Torino, Torino, Italy

88: Also at Bethel University, St. Paul, Minneapolis, U.S.A.

89: Also at Karamanoğlu Mehmetbey University, Karaman, Turkey

90: Also at Ain Shams University, Cairo, Egypt

91: Also at Bingol University, Bingol, Turkey

92: Also at Georgian Technical University, Tbilisi, Georgia

93: Also at Sinop University, Sinop, Turkey

94: Also at Erciyes University, Kayseri, Turkey

95: Also at Institute of Modern Physics and Key Laboratory of Nuclear Physics and Ion-beam Application (MOE) — Fudan University, Shanghai, China

96: Also at Texas A\&M University at Qatar, Doha, Qatar

97: Also at Kyungpook National University, Daegu, Korea 